hep-th/0002131

DUK-CGTP-00-04, IASSNS-HEP-99/117

\title{
The Structure of the D0-D4 Bound State
}

\author{
Savdeep Sethi*1 and Mark Stern ${ }^{\dagger 2}$ \\ * School of Natural Sciences, Institute for Advanced Study, Princeton, NJ 08540, USA \\ $\dagger$ Department of Mathematics, Duke University, Durham, NC 27r06, USA
}

\begin{abstract}
We derive a set of equations for the wavefunction describing the marginal bound state of a single D0-brane with a single D4-brane. These are equations determining the vacuum of an $N=8$ abelian gauge theory with a charged hypermultiplet. We then solve these equations for the most general possible zero-energy solution using a Taylor series. We find that there are an infinite number of such solutions of which only one must be normalizable. We explore the structure of a normalizable solution under the assumption of an asymptotic expansion. Even the leading terms in the asymptotic series, which should reflect the supergravity solution, are unusual. Through the Spin(5) flavor symmetry, the modes which are massive at long distance actually influence the leading behavior. Lastly, we show that the vacuum equations can quite remarkably be reduced to a single equation involving one unknown function. The resulting equation has a surprisingly simple and suggestive form.
\end{abstract}

$2 / 00$

\footnotetext{
1 sethi@sns.ias.edu

2 stern@math.duke.edu
} 


\section{Introduction}

A single D0-brane and a single D4-brane form a marginal bound state [1]. The lowenergy dynamics of a D0-brane in the presence of a D4-brane is described by a quantum mechanical Yang-Mills theory with eight supercharges. The theory has a $U(1)$ vector multiplet coupled to a charged hypermultiplet [2]. With a single D4-brane, there is only a Coulomb branch. The same quantum mechanics appears in the problem of counting $\mathrm{H}$-monopole ground states in the toroidally compactified heterotic string [3]. While the structure of vacuum wavefunctions in marginally bound systems is typically very difficult to analyze, this particular theory has a number of simplifying features. The aim of this paper is to study the vacuum wavefunction of this $0+1$-dimensional gauge theory with eight supercharges.

Our goal is to gain insight into a number of issues. For example, how do we go about uncovering the structure of threshold wavefunctions? It is not even clear how to formulate reasonable questions about a system as complex as the quantum mechanics describing many D0-branes. Another major issue is how the full quantum mechanics resolves the singularity of the moduli space metric. The vector multiplet contains five scalars $x^{\mu}$. For large $r=|x|$, the effective action describing the Coulomb branch dynamics should be a reasonable description of the physics 四. The metric on the Coulomb branch is protected by supersymmetry [5] and takes the form,

$$
d s^{2}=\left(\frac{1}{g^{2}}+\frac{1}{r^{3}}\right)(d x)^{2},
$$

where $g^{2}$ is the Yang-Mills coupling constant. We can express $g^{2}$ in terms of the type IIA string scale $M_{s}$ and coupling constant $g_{s}$,

$$
g^{2}=g_{s} M_{s}^{3} .
$$

This is the only scale in the theory. In the following sections, we set $g^{2}=1$ for simplicity. The tube-like metric (1.1) has a singularity at $r=0$ which is resolved by the full quantum mechanics. Metrics with a similar structure appear in D1-D5 systems.

In the following section, we present the supercharges and describe the symmetries of the problem. The flavor symmetry is $\operatorname{Spin}(5) \times S U(2)_{R}$, and the unique vacuum is invariant under this symmetry [6]. In section three, we derive the general form of a gauge invariant and flavor invariant wavefunction. A general wavefunction depends on 11 functions of two 
variables, $r$ and $y$. As above, $r$ is a radial coordinate for the 5 scalars $x^{\mu}$ of the vector multiplet. The hypermultiplet has 4 scalars $q_{i}$, which parametrize the massive directions. We take $y=|q|$. We then derive a set of differential equations that any zero energy wavefunction must obey.

In section four, we analyze the implications of these differential equations. We can immediately reduce the number of unknown functions from 11 to 7 . These 7 functions satisfy 14 first order coupled partial differential equations in 2 variables. We point out some intriguing features of these equations: in particular, there is an interesting formal method of reducing the number of functions and equations in which the harmonic oscillator plays a key role. This method for collapsing the system of equations has similarities to the technique used to find non-renormalization theorems [7]. We, however, proceed in a different direction. We solve the 14 equations exactly by Taylor expanding in the $y$ variable. This is an expansion in the massive directions. The first interesting point is that the differential equations alone do not determine the solution. There are actually an infinite number of zero energy solutions. The 'gauge' degrees of freedom are not a finite set of parameters as we might have expected, but an entire function of degrees of freedom.

Essentially, from the perspective of the Taylor series, the problem boils down to solving 2 ordinary differential equations in 3 unknown functions. All the other terms in the wavefunction are determined by these 3 functions of $r$. The condition that must uniquely specify the solution is normalizability. Rather remarkably, this global condition is strong enough to fix an entire arbitrary function. This seems to hint that the kind of principle that should underly $M$ theory involves global rather than local constraints. In a vague sense, this is reminiscent of holography.

In section five, we turn to the practical problem of determining the normalizable solution. It turns out to be difficult to implement the global constraint of normalizability in any nice way. Instead, we expand the solution in an asymptotic series. This is akin to solving the $\mathrm{M}$ theory equations of motion for the geometry of an M5-brane in a derivative expansion. We find that even the leading terms in the solution are unusual. These terms should match a supergravity analysis. However, the structure of these terms is strongly dictated by invariance under the $\operatorname{Spin}(5)$ flavor symmetry. Invariance under $\operatorname{Spin}(5)$ is not a statement about long distance, moduli space physics. It is a statement that requires knowledge of both long and short distance physics because the $\operatorname{Spin}(5)$ generators act on both the massless and massive degrees of freedom. Somewhat contrary to intuition, we 
find that the vacuum state for the massive degrees of freedom at large $r$ is a sum of 3 representations of $\operatorname{Spin}(5)$ : a spherically symmetric $\mathbf{1}$, a $\mathbf{5}$ and a $\mathbf{1 4 .}$

The leading terms in the bound state wavefunction $\Psi$ have the form,

$$
\Psi \sim \frac{1}{r^{3}} e^{-\frac{r y^{2}}{2}}\left|b_{1}>+\frac{x^{\mu}}{r^{4}} e^{-\frac{r y^{2}}{2}}\right| b_{2}>^{\mu}+\frac{x^{\mu} x^{\nu}}{r^{5}} e^{-\frac{r y^{2}}{2}} \mid b_{3}>^{\mu \nu},
$$

where the $\mid b_{i}>$ are constructed from fermions. It seems unlikely that this asymptotic form could have been determined from low-energy considerations alone. In this sense, the massive degrees of freedom, through the Spin(5) flavor symmetry, are important even at arbitrarily long distances. The structure of $\Psi$ in (1.2) really begs for an interpretation both in terms of the supergravity solution of the D0-D4 brane [8], and in terms of the DLCQ description of an M5-brane [2] via Matrix theory [9].

We proceed to compute the general form of $\Psi$ in an asymptotic expansion. This takes us well beyond supergravity. The corrections to the leading terms (1.2) take the form of a perturbation series in the coupling constant $g^{2}$. Each correction depends on an a priori unknown constant, and we give a prescription for determining this constant. This amounts to summing up the higher derivative corrections to the supergravity solution for an M5-brane. Is the asymptotic solution actually convergent as $r \rightarrow 0$ ? We know of no nonperturbative effects in the abelian gauge theory that could be relevant at short distances. However, this does not prove that the solution is convergent. It would be interesting to sum up a sufficient number of terms in the asymptotic series to see whether the solution is well-behaved as $r$ becomes small. This would clarify how the singularity in the metric is resolved from the perspective of a derivative expansion.

We note that finding the bound state wavefunction in an asymptotic series is much like trying to understand $M$ theory in a derivative expansion. In section six, we present a quite different reduction of our initial 14 vacuum equations, one that perhaps an $M$ theorist might use. The result is quite incredible. The entire problem reduces to solving a scalar equation of the form,

$$
(\Delta+\vec{B} \cdot \nabla+W) u=0
$$

where $\Delta=\partial_{r}^{2}+\partial_{y}^{2}$ and $u$ is a particular combination of the functions that appear in the bound state wavefunction. The vector field $\vec{B}$ and potential $W$ are rational functions of $r$ and $y$. Equation (1.3) is both simple and highly suggestive. It would be very interesting to find the solution to (1.3) either analytically or numerically. Supergravity and the structure of the derivative expansion should emerge from the asymptotics of the resulting solution 
for the bound state wavefunction. It would also be extremely interesting to generalize this reduction to non-abelian gauge theories. This might possibly help us understand how to define $\mathrm{M}$ theory through Matrix theory [9]. It does not seem too unlikely to us that the ability to 'deprolong' the initial vacuum equations to get (1.3), as described in section six, is tied to supersymmetry and invariance theorems [6].

There are many additional directions to explore. Some of the simplifying features of the D0-D4 system remain when we add more hypermultiplets. However, there will now be a Higgs branch and the zero energy wavefunctions will spread in an interesting way onto the Higgs branch. Turning on $B$-fields makes the gauge theory on the D4-brane noncommutative [10]. Certain choices of $B$-field should change the asymptotic behavior from polynomial decay to exponential decay. It would also be interesting to actually match the asymptotic structure of the bound state wavefunction with higher derivative corrections to the supergravity solution, like those generated by the $R^{4}$ terms [11, 12, 13].

\section{The D0-D4 Quantum Mechanics}

\subsection{The vector multiplet supercharge}

The D0-D4 system is obtained by dimensionally reducing $N=1$ abelian Yang-Mills with a single charged hypermultiplet from six dimensions. The symmetry group consists of the R-symmetries $\operatorname{Spin}(5) \times S U(2)_{R} \sim S p(2) \times S p(1)_{R}$. The Hamiltonian is invariant under the symmetry group while the eight real supercharges transform in the $(\mathbf{4}, \mathbf{2})$ representation.

Let us begin with the vector multiplet which contains the five scalars $x^{\mu}$ transforming in the $(\mathbf{5}, \mathbf{1})$ of the symmetry group. Let $p^{\mu}$ be the associated canonical momenta obeying,

$$
\left[x^{\mu}, p^{\nu}\right]=i \delta^{\mu \nu} .
$$

Associated to these bosons are eight real fermions $\lambda_{a}$ where $a=1, \ldots, 8$ transforming in the $(\mathbf{4}, \mathbf{2})$ representation of the symmetry group. These fermions obey the usual quantization relation,

$$
\left\{\lambda_{a}, \lambda_{b}\right\}=\delta_{a b}
$$

1 The symmetry group, including both gauge and flavor symmetries, is not globally a product. There are discrete identifications. However, for this analysis we only need the Lie algebra generators so we can ignore global identifications. 
Let $\gamma^{\mu}$ be hermitian real gamma matrices which obey,

$$
\left\{\gamma^{\mu}, \gamma^{\nu}\right\}=2 \delta^{\mu \nu}
$$

An explicit basis for these gamma matrices along with a discussion of the symmetry group action is given in Appendix A.

To write the vector multiplet supercharge, we introduce an auxiliary field $D$ which transforms as $(\mathbf{1}, \mathbf{3})$ under the symmetry group. The $D$-term is independent of $x^{\mu}$. The vector multiplet supercharge is given by:

$$
Q_{a}^{v}=\left(\gamma^{\mu} p^{\mu} \lambda\right)_{a}+D_{a b} \lambda_{b}
$$

The real anti-symmetric matrix $D$ commutes with $\gamma^{\mu}$ because the $S p(1)_{R}$ and $S p(2)$ actions commute. The $D$-term must also satisfy,

$$
D_{a c} D_{b c}=-\delta_{a b}|D|^{2} .
$$

It is then not hard to check that:

$$
\left\{Q_{a}^{v}, Q_{b}^{v}\right\}=\delta_{a b}\left\{p^{2}+|D|^{2}\right\}
$$

Under a symmetry transformation $(U, g) \in S p(2) \times S p(1)_{R}$, we note that

$$
\gamma^{\mu} p^{\mu} \rightarrow U \gamma^{\mu} p^{\mu} U^{-1}, \quad \lambda \rightarrow U g \lambda, \quad D \rightarrow g D g^{-1},
$$

so that

$$
Q^{v} \rightarrow U g Q^{v}
$$

\subsection{The hypermultiplet supercharge}

A hypermultiplet contains four real scalars which we can package into a quaternion $q$ with components $q^{i}$ where $i=1,2,3,4$. This field transforms as $(\mathbf{1}, \mathbf{2})$ under the symmetry group. We again introduce canonical momenta $p_{i}$ satisfying the usual commutation relations.

The hypermultiplet is charged under the $U(1)$ gauge symmetry so we need to deter-

mine how $U(1)$ acts on $q$. The $q^{i}$ parametrize $\mathbb{R}^{4}$ so the symmetry group acting on the hypermultiplet must sit inside,

$$
S O(4) \sim S p(1)_{L} \times S p(1)_{R} .
$$


Gauge transformations and $S p(1)_{R}$ transformations commute. Therefore, the $U(1)$ gauge symmetry sits inside $S p(1)_{L}$. We choose to generate the gauge symmetry by left multiplication on $q$ by $I$. The hermitian generator of gauge transformations on the bosons is then given by,

$$
G_{b}=W_{12}+W_{34},
$$

where

$$
W_{i j}=q_{i} p_{j}-q_{j} p_{i} .
$$

The superpartner to $q$ is a real fermion $\psi_{a}$ with $a=1, \ldots, 8$ satisfying,

$$
\left\{\psi_{a}, \psi_{b}\right\}=\delta_{a b},
$$

and transforming in the $(\mathbf{4}, \mathbf{1})$ representation. In terms of the $s^{j}$ operators given in Appendix $\mathrm{A}$, the free hypermultiplet charge takes the form

$$
Q_{a}^{h_{f}}=s_{a b}^{j} \psi_{b} p_{j} .
$$

Note that since the $s^{j}$ implement right multiplication by a quaternion, they commute with $\gamma^{\mu}$. This free charge obeys the algebra,

$$
\left\{Q_{a}^{h_{f}}, Q_{b}^{h_{f}}\right\}=\delta_{a b} p_{i} p_{i} .
$$

Invariance of (2.12) under the $U(1)$ gauge symmetry requires that

$$
G_{f}=-\frac{i}{2} s_{a b}^{2} \psi_{a} \psi_{b}
$$

generate gauge transformations on $\psi$. The total generator of the $U(1)$ gauge symmetry is then given by,

$$
G=G_{b}+G_{f}=W_{12}+W_{34}-\frac{i}{2} \psi s^{2} \psi .
$$

The full hypermultiplet supercharge $Q^{h}$ also includes couplings to the vector multiplet,

$$
Q_{a}^{h}=s_{a b}^{j} \psi_{b} p_{j}+\left(\gamma^{\mu} s^{j} s^{2}\right)_{a b} \psi_{b} x^{\mu} q_{j} .
$$

The form of the interaction term in (2.15) is fixed up to an overall constant by symmetry. The $s^{2}$ appearing in the interaction term is needed to ensure that $Q^{h}$ is gauge-invariant. The charge obeys the algebra:

$$
\left\{Q_{a}^{h}, Q_{b}^{h}\right\}=\delta_{a b}\left\{p_{i} p_{i}+|x|^{2}|q|^{2}-\frac{i}{4} x^{\mu} \psi \gamma^{\mu} s^{2} \psi\right\}+2 \gamma_{a b}^{\mu} x^{\mu} G .
$$

As we expect, the supersymmetry algebra only closes on the Hamiltonian up to gauge transformations. 


\subsection{The coupled system}

The full supercharge $Q$ is the given by,

$$
Q=Q^{v}+Q^{h},
$$

where we define the $D$-term in the following way: using the components $q^{i}$ and matrices $s^{i}$, we can define a quaternion and its conjugate which act by right multiplication on $\lambda$ :

$$
\begin{aligned}
& q^{R}=s^{1} q^{1}+s^{2} q^{2}+s^{3} q^{3}+s^{4} q^{4} \\
& \bar{q}^{R}=s^{1} q^{1}-s^{2} q^{2}-s^{3} q^{3}-s^{4} q^{4} .
\end{aligned}
$$

We can then write the $D$-term in the form,

$$
D_{a b}=\frac{1}{2}\left(q^{R} s^{2} \bar{q}^{R}\right)_{a b} .
$$

This $D$-term obeys (2.5),

$$
\begin{aligned}
\left(D^{2}\right)_{a b} & =\delta_{a b}|D|^{2} \\
& =\delta_{a b} \frac{1}{4}(q \bar{q})^{2} .
\end{aligned}
$$

The full charge obeys the algebra:

$$
\begin{aligned}
\left\{Q_{a}, Q_{b}\right\} & =\delta_{a b}\left\{p^{\mu} p^{\mu}+|D|^{2}+p_{i} p_{i}+|x|^{2}|q|^{2}+\ldots\right\}+2 \gamma_{a b}^{\mu} x^{\mu} G \\
& =\delta_{a b} 2 H+2 \gamma_{a b}^{\mu} x^{\mu} G .
\end{aligned}
$$

The omitted terms are bilinears in the fermions whose exact form we will not need. The bosonic potential $V$ appearing in (2.19) is given by,

$$
V=|x|^{2}|q|^{2}+\frac{1}{4}|q|^{4} .
$$

Since we have coupled a single hypermultiplet to the $U(1)$ vector multiplet, the only flat direction is $q=0$ and there is no Higgs branch.

\section{Deriving Equations for the Vacuum Wavefunction}

To be consistent with predictions from string duality, there should be a unique vacuum wavefunction for this quantum mechanical gauge theory [3]. An index argument proves that there is at least one normalizable vacuum wavefunction [1]. Coupled with a recent invariance theorem [6], the index result implies that the ground state is unique. 
On quantization, the fermions $\lambda$ and $\psi$ act as gamma matrices on a 256-dimensional spinor wavefunction. A priori, the vacuum wavefunction then consists of 256 complex functions of the 9 bosonic variables $x^{\mu}$ and $q^{i}$. However, we can significantly simplify the problem by using symmetries. First note that any state in the Hilbert space $\mid s>$ must be gauge-invariant,

$$
G \mid s>=0 .
$$

Further, all states can be grouped into representations of the global $S p(2) \times S p(1)_{R}$ symmetry group. The $S p(2)$ is generated by the operators,

$$
T^{\mu \nu}=X^{\mu \nu}-\frac{i}{4} \gamma_{a b}^{\mu \nu}\left(\lambda_{a} \lambda_{b}+\psi_{a} \psi_{b}\right)
$$

where

$$
X^{\mu \nu}=x^{\mu} p^{\nu}-x^{\nu} p^{\mu}
$$

The three generators of $S p(1)_{R}$ correspond to right multiplication by $I, J, K$ and in accord with prior notation, we will denote them by $\tilde{s}^{i}$ :

$$
\begin{aligned}
\tilde{s}^{2} & =W_{12}-W_{34}+\frac{i}{2} \lambda s^{2} \lambda \\
\tilde{s}^{3} & =W_{13}+W_{24}+\frac{i}{2} \lambda s^{3} \lambda \\
\tilde{s}^{4} & =W_{14}-W_{23}+\frac{i}{2} \lambda s^{4} \lambda .
\end{aligned}
$$

The unique ground state $\Psi$ must be invariant under the actions of $Q_{a}, G, T^{\mu \nu}$ and $\tilde{s}^{i}$,

$$
Q_{a} \Psi=G \Psi=T^{\mu \nu} \Psi=\tilde{s}^{i} \Psi=0 .
$$

These constraints are quite powerful; for example, they allow us to replace a differential operator $X^{\mu \nu}$ by an algebraic one $\frac{i}{4} \gamma_{a b}^{\mu \nu}\left(\lambda_{a} \lambda_{b}+\psi_{a} \psi_{b}\right)$. There are multiple ways to derive equations for the vacuum wavefunction. We will describe two approaches which we used to derive these equations.

2 Using two different approaches helped enormously in the search for errors. 


\subsection{Radial coordinates}

In the first approach, we can rewrite the supercharge (2.17) in terms of radial coordinates:

$$
r^{2}=|x|^{2}, \quad y^{2}=|q|^{2}
$$

The charge takes the form,

$$
\begin{aligned}
Q_{a} & =\left(\gamma^{\mu} \lambda\right)_{a} \frac{x^{\mu}}{r} p_{r}+\left(s^{j} \psi\right)_{a} \frac{q^{j}}{y} p_{y}+\left(\gamma^{\mu} \lambda\right)_{a} \frac{x^{\nu}}{r^{2}} X^{\nu \mu}+\left(s^{j} \psi\right)_{a} \frac{q^{k}}{y^{2}} W_{k j} \\
& +\left(\gamma^{\mu} s^{j} s^{2} \psi\right)_{a} x^{\mu} q_{j}+(D \lambda)_{a} \\
& =\left(\gamma^{\mu} \lambda\right)_{a} \frac{x^{\mu}}{r} p_{r}+\left(s^{j} \psi\right)_{a} \frac{q^{j}}{y} p_{y}+M_{a} .
\end{aligned}
$$

We have lumped the angular derivatives and non-derivative terms into the operator $M_{a}$. What is particularly nice about $M_{a}$ is that we can replace all the derivative operators by bilinears in fermions. As we noted before, $S p(2)$ invariance allows us to replace $X^{\nu \mu}$ by

$$
\frac{i}{4} \gamma_{a b}^{\nu \mu}\left(\lambda_{a} \lambda_{b}+\psi_{a} \psi_{b}\right)
$$

However, we can also replace $q^{k} W_{k j}$ by a bilinear in fermions using $S p(1)_{R}$ invariance. Using (3.4), we note that:

$$
\begin{aligned}
q^{k} W_{k 1} & =\frac{i}{2}\left\{q^{2} \lambda s^{2} \lambda+q^{3} \lambda s^{3} \lambda+q^{4} \lambda s^{4} \lambda\right\} \\
q^{k} W_{k 2} & =\frac{i}{2}\left\{q^{4} \lambda s^{3} \lambda-q^{1} \lambda s^{2} \lambda-q^{3} \lambda s^{4} \lambda\right\} \\
q^{k} W_{k 3} & =\frac{i}{2}\left\{q^{2} \lambda s^{4} \lambda-q^{1} \lambda s^{3} \lambda-q^{4} \lambda s^{2} \lambda\right\} \\
q^{k} W_{k 4} & =\frac{i}{2}\left\{q^{3} \lambda s^{2} \lambda-q^{1} \lambda s^{4} \lambda-q^{2} \lambda s^{3} \lambda\right\}
\end{aligned}
$$

Therefore, $M_{a}$ is a completely algebraic operator.

Using the $S p(2)$ symmetry, we can then rotate $x$ to the special point where $x^{1} \neq 0$ and $x^{\mu}=0$ for $\mu>1$. Likewise, we can rotate $q$ using $S p(1)_{R}$ to the point $q^{1} \neq 0$ and $q^{i}=0$ for $i>1$. At this point, $r=\left|x^{1}\right|$ and $y=\left|q^{1}\right|$. Since all angular derivatives in $Q_{a}$ are replaced by algebraic operators, there is no difficulty in restricting $\Psi$ to this point. The question of determining $\Psi$ at this point then reduces to finding coupled differential equations in two variables. The form of $\Psi$ at an arbitrary choice of $x$ and $q$ can then be obtained by applying the rotation generators (3.2) and (3.4). 


\subsection{Symmetries and the fermion Hilbert space}

In the second approach which we will use for the rest of the paper, we will first solve the invariance conditions explicitly for the most general possible invariant wavefunction. As we will show, the most general wavefunction depends on 11 functions of $r$ and $y$. We will then derive coupled equations for these functions from the requirement that the wavefunction have zero energy.

The first step is to construct the fermion Hilbert space. We need to complexify our real fermions and build a Fock space:

$$
\begin{array}{ll}
\sqrt{2} d u_{1}=\lambda_{1}+i \lambda_{2} & \sqrt{2} d v_{1}=\psi_{1}+i \psi_{2}, \\
\sqrt{2} d u_{2}=\lambda_{3}-i \lambda_{4} & \sqrt{2} d v_{2}=\psi_{3}-i \psi_{4}, \\
\sqrt{2} d u_{3}=\lambda_{5}+i \lambda_{6} & \sqrt{2} d v_{3}=\psi_{5}+i \psi_{6}, \\
\sqrt{2} d u_{4}=\lambda_{7}-i \lambda_{8} & \sqrt{2} d v_{4}=\psi_{7}-i \psi_{8} .
\end{array}
$$

It is natural to think of $d u_{a}$ and $d v_{a}$ as one-forms obeying the relation,

$$
\left\{d u_{a}, d u_{b}^{*}\right\}=\delta_{a b} \quad\left\{d v_{a}, d v_{b}^{*}\right\}=\delta_{a b},
$$

where $a=1, \ldots, 4$. Wavefunctions in the Hilbert space are then $(p, q)$ forms where $p$ and $q$ are the $d u_{a}$ and $d v_{a}$ degrees, respectively. We choose the Fock vacuum or $(0,0)$ form to satisfy,

$$
d u_{a}^{*}\left|0>=d v_{a}^{*}\right| 0>=0
$$

Note that the complex conjugate of a $(p, q)$-form is a $(4-p, 4-q)$-form. If the ground state is unique then it is bosonic so the form degree must be even.

With our choice of complexification (3.7), the $S p(2)$ generators $T^{\mu \nu}$ acting on forms preserve degree. Actually, the generators preserve $p$ and $q$ separately so a $(p, q)$ form is mapped to a $(p, q)$ form. The $S p(2)$ generators naturally split into commuting generators for an $S p(2)_{b}$ acting on bosons and an $S p(2)_{f}$ acting on fermions. In turn, the $S p(2)_{f}$ splits into an $S p(2)_{f_{p}}$ acting on $d u$ with generators,

$$
-\frac{i}{4} \sum_{a, b} \gamma_{a b}^{\nu \mu} \lambda_{a} \lambda_{b},
$$

and an $S p(2)_{f_{q}}$ acting on $d v$ with generators,

$$
-\frac{i}{4} \sum_{a, b} \gamma_{a b}^{\nu \mu} \psi_{a} \psi_{b}
$$


We can now employ some group theory to see how the various 128 bosonic forms transform under $S p(2)_{f}$. Let us start with the $(p, 0)$ forms which appear in the following representations:

\begin{tabular}{|c|c|}
\hline$p$ & $S p(2)_{f}$ rep. \\
\hline 0 & $\mathbf{1}$ \\
1 & $\mathbf{4}$ \\
2 & $\mathbf{5} \oplus \mathbf{1}$ \\
3 & $\mathbf{4}$ \\
4 & $\mathbf{1}$ \\
\hline
\end{tabular}

We wedge the (odd) even $(p, 0)$ forms with the (odd) even $(0, q)$ forms to get the 128 bosonic forms. The following representations appear from wedging even forms with even forms,

$$
(\mathbf{1})^{10} \oplus(\mathbf{5})^{6} \oplus \mathbf{1 0} \oplus \mathbf{1 4}
$$

while from wedging odd forms with odd forms, we find:

$$
(\mathbf{1})^{4} \oplus(\mathbf{5})^{4} \oplus(\mathbf{1 0})^{4}
$$

We can immediately discard forms transforming in the $\mathbf{1 0}$ representation. A tensor say $a^{\mu \nu}$ transforming in the $\mathbf{1 0}$ is antisymmetric in $\mu, \nu$ so contraction with $x^{\mu} x^{\nu}$ to get a singlet of the full $S p(2)$ gives zero.

Let us now constrain our Hilbert space further by imposing invariance under $S p(1)_{R}$ and the gauge symmetry. We can rewrite the generators (3.4) in terms of our complex fermions:

$$
\begin{aligned}
& \tilde{s}^{2}=W_{12}-W_{34}+\sum_{a} d u_{a} d u_{a}^{*}-2 \\
& \tilde{s}^{3}=W_{13}+W_{24}-i\left(d u_{1} d u_{2}+d u_{1}^{*} d u_{2}^{*}+d u_{3} d u_{4}+d u_{3}^{*} d u_{4}^{*}\right) \\
& \tilde{s}^{4}=W_{14}-W_{23}+\left(d u_{1} d u_{2}-d u_{1}^{*} d u_{2}^{*}+d u_{3} d u_{4}-d u_{3}^{*} d u_{4}^{*}\right) .
\end{aligned}
$$

Likewise for the gauge symmetry,

$$
G=W_{12}+W_{34}-\sum_{a} d v_{a} d v_{a}^{*}+2
$$

Note that the operator $W_{i j}$ has eigenvalues $n$ and $-n$ with corresponding eigenfunctions,

$$
z_{i j}^{n}=\left(q_{i}+i q_{j}\right)^{n}, \quad \bar{z}_{i j}^{n}=\left(q_{i}-i q_{j}\right)^{n}
$$


What does invariance under (3.8) and (3.9) imply? By taking the sum and difference of $G$ and $\tilde{s}^{2}$, we see that we should restrict to $(p, q)$ forms

$$
\left(z_{12}\right)^{\frac{q-p}{2}}\left(z_{34}\right)^{\frac{(p+q)}{2}-2} \mid p, q>
$$

which can be multiplied by a function of both $\left|z_{12}\right|^{2}$ and $\left|z_{34}\right|^{2}$. The remaining two generators $\tilde{s}^{3}$ and $\tilde{s}^{4}$ change the value of $p$. It is natural to study the complex combinations $\tilde{s}^{3}-i \tilde{s}^{4}$ and $\tilde{s}^{3}+i \tilde{s}^{4}$, which raise and lower the value of $p$ :

$$
\begin{aligned}
& s^{+}=\tilde{s}^{3}-i \tilde{s}^{4}=z_{12} p_{34}-\bar{z}_{34} \bar{p}_{12}-2 i\left(d u_{1} d u_{2}+d u_{3} d u_{4}\right) \\
& s^{-}=\tilde{s}^{3}+i \tilde{s}^{4}=\bar{z}_{12} \bar{p}_{34}-z_{34} p_{12}-2 i\left(d u_{1}^{*} d u_{2}^{*}+d u_{3}^{*} d u_{4}^{*}\right),
\end{aligned}
$$

where $p_{i j}=p_{i}-i p_{j}$ and $\bar{p}_{i j}=p_{i}+i p_{j}$.

Again the generators of $S p(1)_{R}$ split into an $S p(1)_{b}$ acting on bosons and an $S p(1)_{f}$ acting on fermions. It is easy to see how the $(p, q)$-forms fall into representations of $S p(1)_{f}$. The three singlets under $S p(2)_{f_{p}}$ denoted $|0, q>| 2,, q>_{\mathbf{1}_{p}}, \mid 4, q>$ transform in the $\mathbf{3}$ of $S p(1)_{f}$. For the choice $q=0,4$, we can construct one singlet under the full $S p(1)_{R}$ which can be multiplied by an arbitrary function of $y$. For the case $q=2$, we can construct two singlets under $S p(1)_{R}$ by tensoring with either the $\mathbf{1}$ or the $\mathbf{5}$ of $S p(2)_{f_{q}}$. Let us denote the $\mathbf{5}$ of $S p(2)_{f_{q}}$ by $\mid 2>_{\mathbf{5}_{q}}^{\mu}$. The explicit $S p(1)_{R}$ singlets are then given by the forms,

$$
\begin{aligned}
& \left\{\bar{z}_{34}^{2}+\bar{z}_{12} \bar{z}_{34}\left(d u_{1} d u_{2}+d u_{3} d u_{4}\right)+\bar{z}_{12}^{2} d u_{1} d u_{2} d u_{3} d u_{4}\right\} \mid 0>, \\
& \left\{z_{12} \bar{z}_{34}+\frac{1}{2}\left(\left|z_{12}\right|^{2}-\left|z_{34}\right|^{2}\right)\left(d u_{1} d u_{2}+d u_{3} d u_{4}\right)-\bar{z}_{12} z_{34} d u_{1} d u_{2} d u_{3} d u_{4}\right\} \times \\
& \left(d v_{1} d v_{2}+d v_{3} d v_{4}\right) \mid 0> \\
& \left\{z_{12} \bar{z}_{34}+\frac{1}{2}\left(\left|z_{12}\right|^{2}-\left|z_{34}\right|^{2}\right)\left(d u_{1} d u_{2}+d u_{3} d u_{4}\right)-\bar{z}_{12} z_{34} d u_{1} d u_{2} d u_{3} d u_{4}\right\} \mid 2>_{\mathbf{5}_{q}}^{\mu}, \\
& \left\{z_{12}^{2}-z_{12} z_{34}\left(d u_{1} d u_{2}+d u_{3} d u_{4}\right)+z_{34}^{2} d u_{1} d u_{2} d u_{3} d u_{4}\right\} d v_{1} d v_{2} d v_{3} d v_{4} \mid 0>.
\end{aligned}
$$

The $\mathbf{5}$ of $S p(2)_{f_{p}}$ denoted $\mid 2>_{\mathbf{5}_{p}}^{\mu}$ decomposes into five singlets under $S p(1)_{f}$. The form $\mid 2>_{\mathbf{5}_{p}}$ can therefore only appear with a function of $y$. To satisfy the constraint (3.10), we must then tensor $\mid 2>_{\mathbf{5}_{p}}^{\mu}$ with a $q=2$ form constructed from $d v$. The two choices are either the $\mathbf{1}$ or the $\mathbf{5}$ of $S p(2)_{f_{q}}$. This gives three additional possibilities denoted,

$$
\left|2,2>_{\mathbf{1}}, \quad\right| 2,2>_{\mathbf{5}}^{\mu}, \quad \mid 2,2>_{\mathbf{1 4}}^{\mu \nu},
$$

where the subscript denotes the representation under the full $S p(2)_{f}$. The construction of these forms is described in Appendix B. 
Lastly, we need to consider the case of odd $p$. The $\mid 1>_{\boldsymbol{4}_{p}}$ and $\mid 3>_{\boldsymbol{4}_{p}}$ forms combine to form a doublet under $S p(1)_{f}$. By tensoring with either the $\mid 1>_{\mathbf{4}_{q}}$ or the $\mid 3>_{\mathbf{4}_{q}}$ forms, we can construct the following four $S p(1)_{R}$ invariants:

$$
\begin{aligned}
& \left\{z_{12}-z_{34}\left(d u_{1} d u_{2}+d u_{3} d u_{4}\right)\right\} \mid 1,3>_{\mathbf{1}} \\
& \left\{z_{12}-z_{34}\left(d u_{1} d u_{2}+d u_{3} d u_{4}\right)\right\} \mid 1,3>_{\mathbf{5}}^{\mu} \\
& \left\{\bar{z}_{34}+\bar{z}_{12}\left(d u_{1} d u_{2}+d u_{3} d u_{4}\right)\right\} \mid 1,1>_{\mathbf{1}} \\
& \left\{\bar{z}_{34}+\bar{z}_{12}\left(d u_{1} d u_{2}+d u_{3} d u_{4}\right)\right\} \mid 1,1>_{\mathbf{5}}^{\mu} .
\end{aligned}
$$

Again the subscript denotes the representation under the full $S p(2)_{f}$. After imposing all the invariance constraints, we are therefore left with 11 complex functions $f_{i}=f_{i}(r, y)$ appearing in the following way:

$$
\begin{gathered}
f_{1}\left|0,0>=f_{1}\left\{\bar{z}_{34}^{2}+\bar{z}_{12} \bar{z}_{34}\left(d u_{1} d u_{2}+d u_{3} d u_{4}\right)+\bar{z}_{12}^{2} d u_{1} d u_{2} d u_{3} d u_{4}\right\}\right| 0> \\
f_{2} \mid 0,4>=f_{2}\left\{z_{12}^{2}-z_{12} z_{34}\left(d u_{1} d u_{2}+d u_{3} d u_{4}\right)+z_{34}^{2} d u_{1} d u_{2} d u_{3} d u_{4}\right\} \\
\times d v_{1} d v_{2} d v_{3} d v_{4} \mid 0> \\
f_{3}\left|2,2>_{\mathbf{1}}, \quad f_{4} x^{\mu}\right| 2,2>_{\mathbf{5}}^{\mu}, \quad f_{5} x^{\mu} x^{\nu} \mid 2,2>_{\mathbf{1 4}}^{\mu \nu}, \\
f_{6}\left|1,3>=f_{6}\left\{z_{12}-z_{34}\left(d u_{1} d u_{2}+d u_{3} d u_{4}\right)\right\}\right| 1,3>_{\mathbf{1}} \\
f_{7}\left|1,1>=f_{7}\left\{\bar{z}_{34}+\bar{z}_{12}\left(d u_{1} d u_{2}+d u_{3} d u_{4}\right)\right\}\right| 1,1>_{\mathbf{1}} \\
f_{8} x^{\mu}\left|1,3>^{\mu}=f_{8} x^{\mu}\left\{z_{12}-z_{34}\left(d u_{1} d u_{2}+d u_{3} d u_{4}\right)\right\}\right| 1,3>_{\mathbf{5}}^{\mu} \\
f_{9} x^{\mu}\left|1,1>^{\mu}=f_{9} x^{\mu}\left\{\bar{z}_{34}+\bar{z}_{12}\left(d u_{1} d u_{2}+d u_{3} d u_{4}\right)\right\}\right| 1,1>_{\mathbf{5}}^{\mu} \\
f_{10} \mid 0,2>=f_{10}\left\{z_{12} \bar{z}_{34}+\frac{1}{2}\left(\left|z_{12}\right|^{2}-\left|z_{34}\right|^{2}\right)\left(d u_{1} d u_{2}+d u_{3} d u_{4}\right)\right. \\
\left.-\bar{z}_{12} z_{34} d u_{1} d u_{2} d u_{3} d u_{4}\right\}\left(d v_{1} d v_{2}+d v_{3} d v_{4}\right) \mid 0> \\
f_{11} x^{\mu} \mid 0,2>^{\mu}=f_{11} x^{\mu}\left\{z_{12} \bar{z}_{34}+\frac{1}{2}\left(\left|z_{12}\right|^{2}-\left|z_{34}\right|^{2}\right)\left(d u_{1} d u_{2}+d u_{3} d u_{4}\right)\right. \\
\left.-\bar{z}_{12} z_{34} d u_{1} d u_{2} d u_{3} d u_{4}\right\} \mid 2>_{\mathbf{5}_{q}}^{\mu} .
\end{gathered}
$$

Our choice of normalization in constructing these forms is described in Appendix C. We take the ground state $\Psi$ to be the sum of these eleven forms. 


\subsection{Dynamical constraints}

What remains is to determine the consequences of the eight equations,

$$
Q_{a} \Psi=0
$$

First note that each term in $Q_{a}$ can be assigned a parity $( \pm, \pm)$ according to whether it changes the parity of the wavefunction in $(x, q)$ respectively. For example, the term

$$
\left(\gamma^{\mu} s^{j} s^{2} \psi\right)_{a} x^{\mu} q_{j}
$$

has parity $(-,-)$ since it is odd in $x$ and odd in $q$. Likewise, each term in $\Psi$ has a definite parity. We can therefore isolate all terms in $Q_{a} \Psi$ with a definite parity. It is also sufficient to restrict to the case $a=1$ because our wavefunction $\Psi$ is $S p(2)$ invariant, but an $S p(2)$ transformation rotates us from one choice of charge to another.

We can then ask: what combinations give terms with parity $(+,-)$ ? A quick check of $Q_{a}$ acting on the possible forms composing $\Psi$ gives the following equation,

$$
\begin{aligned}
& \left(s^{j} \psi\right)_{a} p_{j}\left\{f_{1}\left|0,0>+f_{10}\right| 0,2>+f_{2}\left|0,4>+f_{3}\right| 2,2>_{\mathbf{1}}+f_{5} x^{\mu} x^{\nu} \mid 2,2>_{\mathbf{1 4}}^{\mu \nu}\right\}+ \\
& \left(\gamma^{\mu} s^{j} s^{2} \psi\right)_{a} x^{\mu} q^{j}\left\{f_{4} x^{\rho}\left|2,2>_{\mathbf{5}}^{\rho}+f_{11} x^{\rho}\right| 0,2>^{\rho}\right\} \\
& +\left(\gamma^{\mu} \lambda\right)_{a} p^{\mu}\left\{f_{8} x^{\rho}\left|1,3>^{\rho}+f_{9} x^{\rho}\right| 1,1>^{\rho}\right\}+(D \lambda)_{a}\left\{f_{6}\left|1,3>+f_{7}\right| 1,1>\right\}=0 .
\end{aligned}
$$

The terms giving $(-,-)$ satisfy:

$$
\begin{aligned}
& \left(\gamma^{\mu} s^{j} s^{2} \psi\right)_{a} x^{\mu} q^{j}\left\{f_{1}\left|0,0>+f_{10}\right| 0,2>+f_{2}\left|0,4>+f_{3}\right| 2,2>_{\mathbf{1}}+\right. \\
& \left.f_{5} x^{\mu} x^{\nu} \mid 2,2>_{\mathbf{1 4}}^{\mu \nu}\right\}+\left(s^{j} \psi\right)_{a} p_{j}\left\{f_{4} x^{\rho}\left|2,2>_{\mathbf{5}}^{\rho}+f_{11} x^{\rho}\right| 0,2>^{\rho}\right\} \\
& +(D \lambda)_{a}\left\{f_{8} x^{\rho}\left|1,3>^{\rho}+f_{9} x^{\rho}\right| 1,1>^{\rho}\right\}+\left(\gamma^{\mu} \lambda\right)_{a} p^{\mu}\left\{f_{6}\left|1,3>+f_{7}\right| 1,1>\right\}=0 .
\end{aligned}
$$

From $(-,+)$, we find:

$$
\begin{aligned}
& \left(\gamma^{\mu} \lambda\right)_{a} p^{\mu}\left\{f_{1}\left|0,0>+f_{10}\right| 0,2>+f_{2}\left|0,4>+f_{3}\right| 2,2>_{\mathbf{1}}+f_{5} x^{\mu} x^{\nu} \mid 2,2>_{\mathbf{1 4}}^{\mu \nu}\right\}+ \\
& (D \lambda)_{a}\left\{f_{4} x^{\rho}\left|2,2>_{\mathbf{5}}^{\rho}+f_{11} x^{\rho}\right| 0,2>^{\rho}\right\}+\left(s^{j} \psi\right)_{a} p_{j}\left\{f_{8} x^{\rho}\left|1,3>^{\rho}+f_{9} x^{\rho}\right| 1,1>^{\rho}\right\} \\
& +\left(\gamma^{\mu} s^{j} s^{2} \psi\right)_{a} x^{\mu} q^{j}\left\{f_{6}\left|1,3>+f_{7}\right| 1,1>\right\}=0 .
\end{aligned}
$$

The last equation follows from considering the $(+,+)$ terms,

$$
\begin{aligned}
& (D \lambda)_{a}\left\{f_{1}\left|0,0>+f_{10}\right| 0,2>+f_{2}\left|0,4>+f_{3}\right| 2,2>_{\mathbf{1}}+f_{5} x^{\mu} x^{\nu} \mid 2,2>_{\mathbf{1 4}}^{\mu \nu}\right\}+ \\
& \left(\gamma^{\mu} \lambda\right)_{a} p^{\mu}\left\{f_{4} x^{\rho}\left|2,2>_{\mathbf{5}}^{\rho}+f_{11} x^{\rho}\right| 0,2>^{\rho}\right\}+ \\
& \left(\gamma^{\mu} s^{j} s^{2} \psi\right)_{a} x^{\mu} q^{j}\left\{f_{8} x^{\rho}\left|1,3>^{\rho}+f_{9} x^{\rho}\right| 1,1>^{\rho}\right\}+ \\
& \left(s^{j} \psi\right)_{a} p_{j}\left\{f_{6}\left|1,3>+f_{7}\right| 1,1>\right\}=0 .
\end{aligned}
$$


In each equation, we set $a=1$ as a first simplification. After evaluating angular derivatives, we are free to rotate $q$ using $S p(1)_{R}$ so that $q^{1} \neq 0$ and $q^{i}=0$ for $i>1$. In a similar way, we can consider the point $x^{1} \neq 0$ with $x^{\mu}=0$ for $\mu>1$ after evaluating the $x$ angular derivatives. With this choice of coordinates, $y=\left|q^{1}\right|$ and $r=\left|x^{1}\right|$.

The first set of equations relate $f_{1}, f_{7}, f_{9}, f_{10}$ and $f_{11}$. These follow from considering the $(4,1)$ forms and the $(3,0)$ forms in $(3.21),(3.22)$ and $(3.23),(3.24)$ respectively:

$$
\begin{aligned}
& r \frac{\partial f_{9}}{\partial r}-y \frac{\partial f_{1}}{\partial y}-\frac{y^{2}}{2} f_{7}+5 f_{9}-4 f_{1}+2 f_{10}=0, \\
& \frac{\partial f_{7}}{\partial r}+r y^{2}\left\{f_{1}-\frac{f_{9}}{2}\right\}+2 r f_{11}=0, \\
& r \frac{\partial f_{9}}{\partial y}+y \frac{\partial f_{1}}{\partial r}+r y f_{7}=0, \\
& \frac{\partial f_{7}}{\partial y}+r^{2} y f_{9}+\frac{y^{3}}{2} f_{1}=0 .
\end{aligned}
$$

Likewise, by considering the $(0,3)$ forms in $(3.21),(3.22)$ and the $(1,4)$ forms in $(3.23)$, $(3.24)$, we find the following equations relating $f_{2}, f_{6}, f_{8}, f_{10}$ and $f_{11}$ :

$$
\begin{aligned}
& r \frac{\partial f_{8}}{\partial r}+y \frac{\partial f_{2}}{\partial y}+\frac{y^{2}}{2} f_{6}+5 f_{8}+4 f_{2}-2 f_{10}=0, \\
& \frac{\partial f_{6}}{\partial r}+r y^{2}\left\{f_{2}+\frac{f_{8}}{2}\right\}+2 r f_{11}=0, \\
& y \frac{\partial f_{2}}{\partial r}-r \frac{\partial f_{8}}{\partial y}+r y f_{6}=0, \\
& \frac{\partial f_{6}}{\partial y}-r^{2} y f_{8}+\frac{y^{3}}{2} f_{2}=0 .
\end{aligned}
$$

Note that equations (3.26) are the same as (3.25) under the identification:

$$
f_{2} \rightarrow f_{1} \quad f_{6} \rightarrow f_{7} \quad f_{8} \rightarrow-f_{9} .
$$

It is easy to check there are no non-vanishing $(0,1),(4,3)$ and $(1,0),(3,4)$ forms in (3.21), (3.22) and (3.23), (3.24). This leaves equations coming from forms with degree $(2,1),(2,3)$ in $(3.21)$ and $(3.22)$, and $(1,2),(3,2)$ in $(3.23)$ and $(3.24)$. Let us start by considering the $(2,1)$ parts of $(3.21)$ which give the following constraints,

$$
\begin{aligned}
& f_{10}+\frac{y}{2} \frac{\partial f_{10}}{\partial y}-2 f_{1}+4 f_{9}+r^{2} f_{4}+\frac{1}{y}\left(\frac{\partial f_{3}}{\partial y}+\frac{4}{5} r^{2} \frac{\partial f_{5}}{\partial y}\right)+\frac{y^{2}}{2} r^{2} f_{11}=0, \\
& f_{10}+\frac{y}{2} \frac{\partial f_{10}}{\partial y}-2 f_{1}-r^{2} f_{4}+r \frac{\partial f_{9}}{\partial r}+f_{9}-\frac{1}{y}\left(\frac{\partial f_{3}}{\partial y}+\frac{4}{5} r^{2} \frac{\partial f_{5}}{\partial y}\right) \\
& +\frac{y^{2}}{2} f_{7}+\frac{y^{2}}{2} r^{2} f_{11}=0, \\
& \frac{2}{5} \frac{r^{2}}{y} \frac{\partial f_{5}}{\partial y}-\frac{2}{y} \frac{\partial f_{3}}{\partial y}-r \frac{\partial f_{9}}{\partial r}-3 f_{9}+\frac{y^{2}}{2} f_{7}=0 .
\end{aligned}
$$


The $(2,3)$ terms give the equations,

$$
\begin{aligned}
& f_{10}+\frac{y}{2} \frac{\partial f_{10}}{\partial y}-2 f_{2}-4 f_{8}+r^{2} f_{4}+\frac{1}{y}\left(\frac{\partial f_{3}}{\partial y}+\frac{4}{5} r^{2} \frac{\partial f_{5}}{\partial y}\right)+\frac{y^{2}}{2} r^{2} f_{11}=0, \\
& f_{10}+\frac{y}{2} \frac{\partial f_{10}}{\partial y}-2 f_{2}-r \frac{\partial f_{8}}{\partial r}-f_{8}-r^{2} f_{4}-\frac{1}{y}\left(\frac{\partial f_{3}}{\partial y}+\frac{4}{5} r^{2} \frac{\partial f_{5}}{\partial y}\right) \\
& +\frac{y^{2}}{2} f_{6}+\frac{y^{2}}{2} r^{2} f_{11}=0, \\
& \frac{2}{5} \frac{r^{2}}{y} \frac{\partial f_{5}}{\partial y}-\frac{2}{y} \frac{\partial f_{3}}{\partial y}+r \frac{\partial f_{8}}{\partial r}+3 f_{8}+\frac{y^{2}}{2} f_{6}=0 .
\end{aligned}
$$

Note that equations (3.29) are the same as (3.28) under the identification (3.27). This is a nice check that the equations are correct.

We next need the $(2,1)$ parts of $(3.22)$ which give the relations,

$$
\begin{aligned}
& \frac{y^{2}}{2} f_{10}+f_{3}+\frac{4}{5} r^{2} f_{5}+\frac{1}{y} \frac{\partial f_{4}}{\partial y}+\frac{y}{2} \frac{\partial f_{11}}{\partial y}+f_{11}=0 \\
& \frac{y^{2}}{2} f_{10}-f_{3}-\frac{4}{5} r^{2} f_{5}-\frac{1}{y} \frac{\partial f_{4}}{\partial y}+\frac{1}{r} \frac{\partial f_{7}}{\partial r}+\frac{y^{2}}{2} f_{9}+\frac{y}{2} \frac{\partial f_{11}}{\partial y}+f_{11}=0 \\
& 2 f_{3}-\frac{2}{5} r^{2} f_{5}-\frac{1}{r} \frac{\partial f_{7}}{\partial r}+\frac{y^{2}}{2} f_{9}=0 .
\end{aligned}
$$

From the $(2,3)$ components, we find the equations:

$$
\begin{aligned}
& \frac{y^{2}}{2} f_{10}+f_{3}+\frac{4}{5} r^{2} f_{5}+\frac{1}{y} \frac{\partial f_{4}}{\partial y}+\frac{y}{2} \frac{\partial f_{11}}{\partial y}+f_{11}=0 \\
& \frac{y^{2}}{2} f_{10}-f_{3}-\frac{4}{5} r^{2} f_{5}-\frac{1}{y} \frac{\partial f_{4}}{\partial y}+\frac{1}{r} \frac{\partial f_{6}}{\partial r}-\frac{y^{2}}{2} f_{8}+\frac{y}{2} \frac{\partial f_{11}}{\partial y}+f_{11}=0 \\
& 2 f_{3}-\frac{2}{5} r^{2} f_{5}-\frac{1}{r} \frac{\partial f_{6}}{\partial r}-\frac{y^{2}}{2} f_{8}=0 .
\end{aligned}
$$

These equations are again consistent with (3.27).

We now turn to the $(1,2)$ parts of $(3.23)$ which imply that,

$$
\begin{aligned}
& \frac{y^{2}}{2 r} \frac{\partial f_{10}}{\partial r}+\frac{1}{r} \frac{\partial f_{3}}{\partial r}+\frac{4}{5} r \frac{\partial f_{5}}{\partial r}+\frac{28}{5} f_{5}+\frac{1}{2} y^{2} f_{4}+2 f_{9}+\frac{y^{4}}{4} f_{11}=0, \\
& \frac{y^{2}}{2 r} \frac{\partial f_{10}}{\partial r}-\frac{1}{r} \frac{\partial f_{3}}{\partial r}-\frac{4}{5} r \frac{\partial f_{5}}{\partial r}-\frac{28}{5} f_{5}+\frac{1}{2} y^{2} f_{4}+y^{2} f_{6} \\
& +y \frac{\partial f_{8}}{\partial y}+2 f_{8}-\frac{y^{4}}{4} f_{11}=0, \\
& \frac{2}{r} \frac{\partial f_{3}}{\partial r}-\frac{2}{5} r \frac{\partial f_{5}}{\partial r}-\frac{14}{5} f_{5}-y^{2} f_{6}+y \frac{\partial f_{8}}{\partial y}+2 f_{8}-2 f_{9}=0 .
\end{aligned}
$$


The $(3,2)$ forms give the following set of equations:

$$
\begin{aligned}
& \frac{y^{2}}{2 r} \frac{\partial f_{10}}{\partial r}+\frac{1}{r} \frac{\partial f_{3}}{\partial r}+\frac{4}{5} r \frac{\partial f_{5}}{\partial r}+\frac{28}{5} f_{5}+\frac{1}{2} y^{2} f_{4}-2 f_{8}+\frac{y^{4}}{4} f_{11}=0 \\
& \frac{y^{2}}{2 r} \frac{\partial f_{10}}{\partial r}-\frac{1}{r} \frac{\partial f_{3}}{\partial r}-\frac{4}{5} r \frac{\partial f_{5}}{\partial r}-\frac{28}{5} f_{5}+\frac{1}{2} y^{2} f_{4}+y^{2} f_{7}-y \frac{\partial f_{9}}{\partial y}- \\
& 2 f_{9}-\frac{y^{4}}{4} f_{11}=0, \\
& \frac{2}{r} \frac{\partial f_{3}}{\partial r}-\frac{2}{5} r \frac{\partial f_{5}}{\partial r}-\frac{14}{5} f_{5}-y^{2} f_{7}-y \frac{\partial f_{9}}{\partial y}-2 f_{9}+2 f_{8}=0 .
\end{aligned}
$$

Again, (3.33) and (3.32) are identical under (3.27).

The $(1,2)$ parts of $(3.24)$ give the following equations,

$$
\begin{aligned}
& \frac{y^{4}}{4} f_{10}+\frac{y^{2}}{2} f_{3}+\frac{2 y^{2} r^{2}}{5} f_{5}+r \frac{\partial f_{4}}{\partial r}+5 f_{4}+2 f_{7}+\frac{y^{2}}{2} f_{11}+\frac{y^{2} r}{2} \frac{\partial f_{11}}{\partial r}=0 \\
& \frac{y^{4}}{4} f_{10}-\frac{y^{2}}{2} f_{3}-\frac{2 y^{2} r^{2}}{5} f_{5}+r \frac{\partial f_{4}}{\partial r}+5 f_{4}+y \frac{\partial f_{6}}{\partial y}+2 f_{6} \\
& +r^{2} y^{2} f_{8}-\frac{y^{2}}{2} f_{11}-\frac{y^{2} r}{2} \frac{\partial f_{11}}{\partial r}=0 \\
& y^{2} f_{3}-\frac{y^{2} r^{2}}{5} f_{5}-y \frac{\partial f_{6}}{\partial y}-2 f_{6}+2 f_{7}+r^{2} y^{2} f_{8}+y^{2} f_{11}=0 .
\end{aligned}
$$

Lastly, the $(3,2)$ parts give the equations:

$$
\begin{aligned}
& \frac{y^{4}}{4} f_{10}+\frac{y^{2}}{2} f_{3}+\frac{2 y^{2} r^{2}}{5} f_{5}+r \frac{\partial f_{4}}{\partial r}+5 f_{4}+2 f_{6}+\frac{y^{2}}{2} f_{11}+\frac{y^{2} r}{2} \frac{\partial f_{11}}{\partial r}=0 \\
& \frac{y^{4}}{4} f_{10}-\frac{y^{2}}{2} f_{3}-\frac{2 y^{2} r^{2}}{5} f_{5}+r \frac{\partial f_{4}}{\partial r}+5 f_{4}+y \frac{\partial f_{7}}{\partial y}+2 f_{7} \\
& -r^{2} y^{2} f_{9}-\frac{y^{2}}{2} f_{11}-\frac{y^{2} r}{2} \frac{\partial f_{11}}{\partial r}=0 \\
& y^{2} f_{3}-\frac{y^{2} r^{2}}{5} f_{5}-y \frac{\partial f_{7}}{\partial y}+2 f_{6}-2 f_{7}-r^{2} y^{2} f_{9}+y^{2} f_{11}=0 .
\end{aligned}
$$

Again, note that (3.34) and (3.35) are identical under the exchange (3.27).

\section{The Structure of the Bound State Wavefunction}

\subsection{Reducing the number of functions}

Initially, we have eleven independent functions obeying a set of coupled differential equations. To make progress, we need to reduce the number of functions in a systematic 
fashion. We can begin to whittle down the number of independent functions in the following way: the difference of the third equations of (3.30) and (3.31) together with (3.25) imply that,

$$
f_{1}=f_{2}
$$

Let us now turn to (3.25). By taking $\partial_{y}$ of the second equation and $\partial_{r}$ of the fourth equation, we find two equations for $\partial_{r y}^{2} f_{7}$. For these equations to be compatible, we require that:

$$
f_{10}-f_{1}+f_{9}+\frac{1}{y} \frac{\partial f_{11}}{\partial y}=0
$$

This equation is actually not a new addition to our list of constraints. It follows from (3.30) and (3.25). The same analysis applied to (3.26) gives,

$$
f_{10}-f_{1}-f_{8}+\frac{1}{y} \frac{\partial f_{11}}{\partial y}=0
$$

These equations together require that,

$$
f_{8}=-f_{9}
$$

The difference of the fourth equations in (3.25) and (3.26) then implies the equivalence,

$$
f_{6}=f_{7}
$$

In this way, we are reduced to eight functions $\left\{f_{1}, f_{3}, f_{4}, f_{5}, f_{7}, f_{9}, f_{10}, f_{11}\right\}$. Half the equations we derived are now redundant since the symmetry (3.27) is an actual identity.

We obtain an algebraic relation between the remaining functions by using the third equation in (3.30) together with (3.25):

$$
f_{3}+\frac{y^{2}}{2} f_{1}-\frac{r^{2}}{5} f_{5}+f_{11}=0
$$

Using the algebraic relation, we can eliminate one function. The remaining 7 functions of 2 variables are constrained by 14 equations, which is the minimal number we could have expected. 


\subsection{A first reduction of the equations}

Let us summarize the equations that describe the bound state. We have taken some simpler linear combinations of the previous equations:

$$
\begin{aligned}
& \text { (1) } r \frac{\partial f_{9}}{\partial r}-y \frac{\partial f_{1}}{\partial y}-\frac{y^{2}}{2} f_{7}+5 f_{9}-4 f_{1}+2 f_{10}=0 \text {, } \\
& \text { (2) } \frac{\partial f_{7}}{\partial r}+r y^{2}\left\{f_{1}-\frac{f_{9}}{2}\right\}+2 r f_{11}=0, \\
& \text { (3) } r \frac{\partial f_{9}}{\partial y}+y \frac{\partial f_{1}}{\partial r}+r y f_{7}=0 \text {, } \\
& \text { (4) } \frac{\partial f_{7}}{\partial y}+r^{2} y f_{9}+\frac{y^{3}}{2} f_{1}=0 \text {, } \\
& \text { (5) } 2 f_{10}+y \frac{\partial f_{10}}{\partial y}-4 f_{1}+5 f_{9}+y^{2} r^{2} f_{11}+r \frac{\partial f_{9}}{\partial r}+\frac{y^{2}}{2} f_{7}=0 \text {, } \\
& \text { (6) } 2 r^{2} y f_{4}-\frac{y^{3}}{2} f_{7}+3 y f_{9}-r y \frac{\partial f_{9}}{\partial r}+2 \frac{\partial f_{3}}{\partial y}+\frac{8}{5} r^{2} \frac{\partial f_{5}}{\partial y}=0 \text {, } \\
& \text { (7) } \frac{2}{5} \frac{r^{2}}{y} \frac{\partial f_{5}}{\partial y}-\frac{2}{y} \frac{\partial f_{3}}{\partial y}-r \frac{\partial f_{9}}{\partial r}-3 f_{9}+\frac{y^{2}}{2} f_{7}=0 \text {, } \\
& \text { (8) } y^{2} f_{10}+\frac{y^{2}}{2} f_{9}+2 f_{11}+y \frac{\partial f_{11}}{\partial y}+\frac{1}{r} \frac{\partial f_{7}}{\partial r}=0 \text {, } \\
& \text { (9) } 2 y f_{3}+\frac{8}{5} r^{2} y f_{5}-\frac{y^{3}}{2} f_{9}+2 \frac{\partial f_{4}}{\partial y}-\frac{y}{r} \frac{\partial f_{7}}{\partial r}=0 \text {, } \\
& \text { (10) } r y\left(f_{4}+f_{7}\right)-r \frac{\partial f_{9}}{\partial y}+y \frac{\partial f_{10}}{\partial r}=0 \text {, } \\
& \text { (11) } \frac{56}{5} r f_{5}-r y^{2} f_{7}+4 r f_{9}+\frac{1}{2} r y^{4} f_{11}+r y \frac{\partial f_{9}}{\partial y}+2 \frac{\partial f_{3}}{\partial r}+\frac{8}{5} r^{2} \frac{\partial f_{5}}{\partial r}=0 \text {, } \\
& \text { (12) } \frac{2}{r} \frac{\partial f_{3}}{\partial r}-\frac{2}{5} r \frac{\partial f_{5}}{\partial r}-\frac{14}{5} f_{5}-y^{2} f_{7}-y \frac{\partial f_{9}}{\partial y}-4 f_{9}=0 \text {, } \\
& 10 f_{4}+4 f_{7}-r^{2} y^{2} f_{9}+\frac{y^{4}}{2} f_{10}+y \frac{\partial f_{7}}{\partial y}+2 r \frac{\partial f_{4}}{\partial r}=0 \\
& y f_{3}+\frac{4}{5} r^{2} y f_{5}+r^{2} y f_{9}+y f_{11}-\frac{\partial f_{7}}{\partial y}+r y \frac{\partial f_{11}}{\partial r}=0 .
\end{aligned}
$$

In these equations, we can remove one function using (4.6). This is a complicated set of coupled equations. To uncover the structure of the wavefunction, let us begin by simplifying as much as possible.

After staring at these equations for sometime, a pair of equations (3) and (4) in (4.7) appear distinguished. At our special point, these equations involve only the $(4,0)$ and $(3,1)$ forms given explicitly in Appendix C. They do not involve any $(2,2)$ forms. These are the 
analogues of the 'top forms' which played a crucial role in proving non-renormalization theorems [7]. We can eliminate $f_{9}$ from these two equations giving,

$$
\begin{aligned}
\left\{-\partial_{y}^{2}+\frac{1}{y} \partial_{y}+r^{2} y^{2}\right\} f_{7} & =y^{2}\left\{1+\frac{y}{2} \partial_{y}-r \partial_{r}\right\} f_{1}, \\
& =y^{2} S\left(f_{1}\right) .
\end{aligned}
$$

Note that if we set the source term $S\left(f_{1}\right)=0$, then the homogeneous solutions for $f_{7}$ are $e^{ \pm r y^{2} / 2}$. The plus sign is not normalizable. However, both solutions suffer from a more serious problem. The ground state wavefunction must be a smooth function. It must have a convergent Taylor series about the origin. This implies that each $f_{i}$ must be function of $r^{2}$ and $y^{2}$ near the origin. The homogeneous solutions alone are therefore ruled out.

What this teaches us is that $f_{7}$ is determined in terms of $f_{1}$. It is convenient to make the following redefinition,

$$
f_{7}=y^{2} \tilde{f}_{7}
$$

Note that $\tilde{f}_{7}$ can have a $1 / y^{2}$ term near the origin. The equation (4.8) now takes the form,

$$
\left\{-\partial_{y}^{2}-\frac{3}{y} \partial_{y}+r^{2} y^{2}\right\} \tilde{f}_{7}=S\left(f_{1}\right) .
$$

The left hand side of (4.9) is the Hamiltonian for a four-dimensional harmonic oscillator! Now there is a very pretty collapse. Equation (4) in (4.7) determines $f_{9}$ in terms of $f_{1}$. Likewise (1) and (2) determine $f_{10}$ and $f_{11}$. Equation (10) determines $f_{4}$, while (14) determines $f_{3}$. The algebraic constraint (4.6) then fixes $f_{5}$. Clearly, there many other ways to collapse the problem. The main point is that all the remaining functions are given in terms of $f_{1}$. This leaves 8 equations which must determine $f_{1}$.

\subsection{Solving for $f_{7}$}

We can now express $f_{7}$ in terms of $f_{1}$ in the following way:

$$
f_{7}=f_{7}^{0}\left(r^{2}\right) e^{-r y^{2} / 2}+y^{2}\left\{-\partial_{y}^{2}-\frac{3}{y} \partial_{y}+r^{2} y^{2}\right\}^{-1} S\left(f_{1}\right) .
$$

The $y=0$ component $f_{7}^{0}$ is determined by requiring that $f_{7}$ be smooth, as we discussed previously. Smoothness of $f_{7}$ also requires that $f_{7}^{0}$ be a function of $r^{2}$. In turn, we can expand $f_{1}$ as follows:

$$
f_{1}=\sum_{n=0}^{\infty} f_{1}^{n}(r) \mid n>
$$


The $\mid n>$ are radial harmonic oscillator eigenstates which obey,

$$
\left\{-\partial_{y}^{2}-\frac{3}{y} \partial_{y}+r^{2} y^{2}\right\}\left|n>=E_{n}\right| n>
$$

where $E_{n}=4(n+1) r$. The construction and properties of these eigenstates are described in Appendix D. These eigenstates have the nice feature that acting on $|n\rangle$, the operators $\partial_{r}, y \partial_{y}$ and $y^{2}$ involve only $|n-1>| n>,, \mid n+1>$. Using the relations from Appendix $\mathrm{D}$, we see that the source term has a beautifully simple form:

$$
S\left(f_{1}\right)=\sum_{n}\left(f_{1}^{n}-r \partial_{r} f_{1}^{n}\right) \mid n>
$$

It is now easy to solve for $f_{7}$ in terms of $\mid n>$,

$$
f_{7}=f_{7}^{0}\left(r^{2}\right)\left|0>+y^{2} \sum_{n=0}^{\infty} \frac{1}{E_{n}}\left(f_{1}^{n}-r \partial_{r} f_{1}^{n}\right)\right| n>\text {. }
$$

We have left $y^{2}$ in (4.13) for later convenience. By considering the coefficient of $y^{2}$ in (4.13) and imposing smoothness, we obtain the following relation:

$$
-\frac{r}{2} f_{7}^{0}+\sum_{n=0}^{\infty} \frac{1}{E_{n}}\left(1-r \partial_{r}\right) f_{1}^{n}=0
$$

Since $f_{7}^{0}$ is non-singular as $r \rightarrow 0$, we obtain the sum rule

$$
\sum_{n=0}^{\infty} \frac{1}{4(n+1)} f_{1}^{n}(0)=0
$$

and the relation:

$$
f_{7}^{0}=-\frac{2}{r^{2}} \sum_{n=0}^{\infty} \frac{1}{4(n+1)}\left(1-r \partial_{r}\right) f_{1}^{n} .
$$

Note that these sum rules may be largely formal since we do not know whether the sums are absolutely convergent.

\subsection{Equations for the physics near the flat directions}

Instead of proceeding to reduce the number of functions, let us take a different tack. The most interesting physics in this problem occurs in a neighbourhood of the flat directions. So let us consider a Taylor expansion about the flat direction $y=0$. This approach 
turns out to be more useful than reducing the number of functions, which increases the complexity of the resulting equations. We expand each $f_{i}$ in the following way,

$$
f_{i}=t_{i}^{0}\left(r^{2}\right)+t_{i}^{2}\left(r^{2}\right) y^{2}+t_{i}^{4}\left(r^{2}\right) y^{4}+\ldots
$$

The algebraic constraint (4.6) together with the equations of (4.7) give the following set of relations on the $t_{i}^{0}$ :

$$
\begin{aligned}
& \text { (1) } t_{3}^{0}-\frac{r^{2}}{5} t_{5}^{0}+t_{11}^{0}=0 \\
& \text { (2) } r \partial_{r} t_{9}^{0}+5 t_{9}^{0}-4 t_{1}^{0}+2 t_{10}^{0}=0 \\
& \text { (3) } \partial_{r} t_{7}^{0}+2 r t_{11}^{0}=0 \\
& \text { (4) } \frac{56}{5} r t_{5}^{0}+4 r t_{9}^{0}+2 \partial_{r} t_{3}^{0}+\frac{8}{5} r^{2} \partial_{r} t_{5}^{0}=0 \\
& \text { (5) } \frac{2}{r} \partial_{r} t_{3}^{0}-\frac{2}{5} r \partial_{r} t_{5}^{0}-\frac{14}{5} t_{5}^{0}-4 t_{9}^{0}=0 \\
& \text { (6) } 10 t_{4}^{0}+4 t_{7}^{0}+2 r \partial_{r} t_{4}^{0}=0 \\
& \text { (7) } t_{4}^{0}+2 t_{7}^{0}+\frac{1}{r} \partial_{r}\left\{t_{1}^{0}+t_{10}^{0}\right\}=0
\end{aligned}
$$

This gives 7 equations for 8 unknown functions. As we might have expected, this is not sufficient to determine the flat direction physics without input from higher $y$ terms. We can similarly derive equations involving only $t_{i}^{2}$ and $t_{i}^{0}$ which are given in Appendix E.

Note the critical observation that we can solve for all $t_{i}^{2}$ in terms of the $t_{i}^{0}$ using 8 of the equations of E.1. There are no new independent functions at order $y^{2}$ in the Taylor expansion. We can express the $t_{i}^{2}$ in terms of the $t_{i}^{0}$ and at most their second derivatives using the first 7 equations and equation (10) of E.1,

$$
\begin{aligned}
& \text { (1) } t_{7}^{2}=-\frac{1}{2} r^{2} t_{9}^{0}, \\
& \text { (2) } t_{9}^{2}=-\frac{1}{2 r}\left\{\partial_{r} t_{1}^{0}+r t_{7}^{0}\right\}, \\
& \text { (3) } t_{11}^{2}=\frac{1}{4}\left\{3 t_{9}^{0}+r \partial_{r} t_{9}^{0}-2 t_{1}^{0}\right\}, \\
& \text { (4) } t_{5}^{2}=-\frac{1}{2 r}\left\{r t_{4}^{0}-\partial_{r} t_{9}^{0}\right\}, \\
& \text { (5) } t_{3}^{2}=\frac{1}{20}\left\{-2 r^{2} t_{4}^{0}-15 t_{9}^{0}-3 r \partial_{r} t_{9}^{0}\right\}, \\
& \text { (6) } t_{4}^{2}=-\frac{1}{20 r}\left\{10 r t_{3}^{0}+8 r^{3} t_{5}^{0}-5 \partial_{r} t_{7}^{0}\right\}, \\
& \text { (7) } t_{10}^{2}=\frac{1}{16}\left\{-6 r^{2} t_{11}^{0}+\frac{4}{r} \partial_{r} t_{1}^{0}+r \partial_{r} t_{7}^{0}+\partial_{r}^{2} t_{1}^{0}\right\}, \\
& \text { (8) } t_{1}^{2}=-\frac{1}{16 r}\left\{8 r t_{7}^{0}+2 r^{3} t_{11}^{0}+4 \partial_{r} t_{1}^{0}+r^{2} \partial_{r} t_{7}^{0}+r \partial_{r}^{2} t_{1}^{0}\right\} .
\end{aligned}
$$


What we need to check is whether any of the remaining 7 equations of $E .1$ are new relations. After some algebra that we will spare the reader, it turns out that all the remaining equations of E.1 are consequences of (4.18). This is not too surprising. The interactions involve order $y^{4}$ terms so we should not be able to completely determine the physics on the flat directions without expanding to higher order in $y$. So let us expand to order $y^{4}$ giving equations relating the $t_{i}^{4}, t_{i}^{2}$ and $t_{i}^{0}$. These equations are again given explicitly in Appendix E.

The equations of E.2 are very similar to $E .1$, except for mixing with some $t_{i}^{0}$ terms through the $y^{3}$ and $y^{4}$ interactions in (4.7). It is easy to see that we can again solve for all the $t_{i}^{4}$ in terms of the $t_{i}^{0}$. It should be clear that all the coefficients of the higher $y^{2 n}$ terms in the Taylor expansion are determined in terms of the $t_{i}^{0}$. The problem is then to determine the $t_{i}^{0}$, and we need one more relation in addition to those of (4.18). Again using the first 7 equations and equation (10) of $E .2$, we can solve for the $t_{i}^{4}$ :

$$
\begin{aligned}
\text { (1) } t_{7}^{4}= & -\frac{1}{4}\left\{r^{2} t_{9}^{2}+\frac{1}{2} t_{1}^{0}\right\}, \\
\text { (2) } t_{9}^{4}= & -\frac{1}{4 r}\left\{\partial_{r} t_{1}^{2}+r t_{7}^{2}\right\}, \\
\text { (3) } t_{11}^{4}= & \frac{1}{16}\left\{8 t_{9}^{2}+2 r \partial_{r} t_{9}^{2}-8 t_{1}^{2}+\frac{1}{r} \partial_{r} t_{1}^{0}\right\}, \\
\text { (4) } t_{5}^{4}= & \frac{1}{16 r^{3}}\left\{r t_{7}^{0}-4 r^{3} t_{4}^{2}+2 r t_{9}^{2}+\partial_{r} t_{1}^{0}+4 r^{2} \partial_{r} t_{9}^{2}\right\}, \\
\text { (5) } t_{3}^{4}= & \frac{1}{80 r}\left\{r t_{7}^{0}-4 r^{3} t_{4}^{2}-38 r t_{9}^{2}-4 \partial_{r} t_{1}^{0}-6 r^{2} \partial_{r} t_{9}^{2}\right\}, \\
\text { (6) } t_{4}^{4}= & \frac{1}{80}\left\{5 t_{9}^{0}-20 t_{3}^{2}-16 r^{2} t_{5}^{2}+\frac{10}{r} \partial_{r} t_{7}^{2}\right\}, \\
\text { (7) } t_{10}^{4}= & \frac{1}{200}\left\{20 r^{2} t_{1}^{0}+40 r^{2} t_{3}^{2}-8 r^{4} t_{5}^{2}-5 t_{7}^{2}+\frac{20}{r} \partial_{r} t_{1}^{2}+5 r \partial_{r} t_{7}^{2}+5 \partial_{r}^{2} t_{1}^{2}\right\}, \\
\text { (8) } t_{1}^{4}= & -\frac{1}{200 r}\left\{-5 r^{3} t_{1}^{0}-10 r^{3} t_{3}^{2}+2 r^{5} t_{5}^{2}+45 r t_{7}^{2}+20 \partial_{r} t_{1}^{2}\right. \\
& \left.+5 r^{2} \partial_{r} t_{7}^{2}+5 r \partial_{r}^{2} t_{1}^{2}\right\} .
\end{aligned}
$$

Note that the expression for $t_{7}^{4}$ agrees with the expression coming from the earlier relation (4.8) that we derived between $f_{1}$ and $f_{7}$. Once again we are left with 7 additional equations. It turns out that these additional equations again give no new relations. After checking higher order Taylor coefficients, we find that there are no further relations. It appears that any choice of $t_{i}^{0}$ satisfying (4.18) give a zero energy solution. However, most of these solutions are not normalizable. 
It is straightforward to derive the general recursion relation for $t_{i}^{n}$ in terms of lower Taylor coefficients:

(1) $t_{7}^{n}=-\frac{1}{n}\left\{r^{2} t_{9}^{n-2}+\frac{1}{2} t_{1}^{n-4}\right\}$,

(2) $t_{9}^{n}=-\frac{1}{n r}\left\{\partial_{r} t_{1}^{n-2}+r t_{7}^{n-2}\right\}$,

(3) $t_{11}^{n}=\frac{1}{2}\left\{\frac{1}{2} t_{9}^{n-2}-t_{1}^{n-2}-\frac{1}{r} \partial_{r} t_{7}^{n}\right\}$,

(4) $t_{5}^{n}=\frac{1}{n}\left\{\frac{1}{r} \partial_{r} t_{9}^{n-2}-t_{4}^{n-2}\right\}$

(5) $t_{3}^{n}=\left\{\frac{r^{2}}{5} t_{5}^{n}-t_{11}^{n}-\frac{1}{2} t_{1}^{n-2}\right\}$,

(6) $t_{4}^{n}=\frac{1}{2 n}\left\{\frac{1}{r} \partial_{r} t_{7}^{n-2}+\frac{1}{2} t_{9}^{n-4}-2 t_{3}^{n-2}-\frac{8}{5} r^{2} t_{5}^{n-2}\right\}$,

(7) $t_{10}^{n}=-\frac{1}{2 n(n+6)}\left\{(n+8) t_{7}^{n-2}+10 n t_{9}^{n}+r^{2}(2 n+8) t_{11}^{n-2}+2 n r \partial_{r} t_{9}^{n}\right\}$,

(8) $t_{1}^{n}=-\frac{1}{n}\left\{t_{7}^{n-2}+n t_{10}^{n}+r^{2} t_{11}^{n-2}\right\}$.

Let us close this discussion of the Taylor expansion by pointing out that the entire Taylor series depends only on 3 of the $t_{i}^{0}$. To see this, let us express (4.18) in a form which will be more convenient for later manipulation,

(1) $t_{5}^{0}=\frac{5}{r^{2}}\left(t_{3}^{0}+t_{11}^{0}\right)$

(2) $t_{11}^{0}=-\frac{1}{2 r} \partial_{r} t_{7}^{0}$

(3) $t_{7}^{0}=-\frac{1}{2}\left(5 t_{4}^{0}+r \partial_{r} t_{4}^{0}\right)$,

(4) $t_{9}^{0}=\frac{5}{6 r} \partial_{r} t_{3}^{0}$

(5) $t_{10}^{0}=2 t_{1}^{0}-\frac{5}{2} t_{9}^{0}-\frac{r}{2} \partial_{r} t_{9}^{0}$

(6) $20 \partial_{r}\left(r^{3} t_{3}^{0}\right)+r\left\{72 \partial_{r}+33 r \partial_{r}^{2}+3 r^{2} \partial_{r}^{3}\right\} t_{4}^{0}=0$,

(7) $\partial_{r} t_{1}^{0}+\frac{1}{48 r^{4}}\left\{-64 r^{5}+72 \partial_{r}-16 r^{6} \partial_{r}-72 r \partial_{r}^{2}\right.$

$\left.+12 r^{2} \partial_{r}^{3}+12 r^{3} \partial_{r}^{4}+r^{4} \partial_{r}^{5}\right\} t_{4}^{0}=0$.

The first 5 equations of (4.22) are definitions of various $t_{i}^{0}$. The last 2 are relations on the 3 independent functions $t_{1}^{0}, t_{3}^{0}$ and $t_{4}^{0}$. 


\section{An Asymptotic Expansion of the Wavefunction}

\subsection{Matching Taylor and oscillator expansions}

It is hard to see how to implement the normalizability condition in a Taylor series. So although we have found the zero energy solution in terms of the $t_{i}^{0}$, we need a practical procedure to construct the $t_{i}^{0}$. We will determine the $t_{i}^{0}$ under the assumption that the bound state wavefunction admit an asymptotic expansion in powers of $1 / r$. The primary motivation for studying the asymptotic expansion is that the asymptotic form should be interpretable in terms of supergravity plus higher derivative corrections. What we do not know is whether the asymptotic expansion converges to the actual bound state wavefunction. This issue is closely related to the following two questions: if we sum the effects of all higher derivative terms in the effective action for this gauge theory, does the result give non-singular physics as $r \rightarrow 0$ ? If we sum the effects of all higher derivative terms beyond supergravity on the spacetime solution for a 5-brane in $\mathrm{M}$ theory, do we find a smooth convergent solution near the 5-brane?

These questions are intrinsically tied to the problem of gauge-fixing the Taylor series solution in a way that results in a normalizable solution. We want to construct the $t_{i}^{0}$ in a useful systematic expansion. The dominant terms in an asymptotic expansion are those that decay polynomially in $1 / r$. We point out that for large $r$, an approximate asymptotic bound state can be constructed in a $1 / r$ expansion using a method described in [14]. An analytic expansion in $1 / r$ near infinity is essentially a perturbative expansion, although not in $g^{2}$ a priori but in $g^{2 / 3}$. A similar technique was used in [15, 16, 17 to further explore the long distance dynamics and the asymptotic structure of the bound state wavefunction for 2 D0-branes. Unfortunately, the effective long distance Hamiltonian has only been constructed to order $1 / r^{2}$, which is the required order for an index computation 14,18. With our knowledge of the Taylor series solution (4.21) for this problem, we can do significantly better than those approximate constructions.

Can there be non-perturbative terms? These are terms which are not visible in a $1 / r$ expansion, like $e^{-r^{2}}$, but which become important as $r$ becomes small. We do not actually know whether there are any such terms, and there are no candidates like instanton configurations that could generate these terms in the abelian gauge theory. This leads the first author to suspect that the analytic expansion in $1 / r$ might well be exact. Nevertheless, we cannot prove that non-perturbative terms are not present. To really rule out such terms, 
we need a technique for finding the bound state solution which is inherently more global than an asymptotic expansion. We shall discuss a more global approach in section 6 .

What is hard to see in the Taylor expansion of section 4 is normalizability in the $y$-direction. This is much easier to see in the oscillator expansion so we will match the two expansions to get control over the question of normalizability. When expanded in a harmonic oscillator basis with frequency proportional to $r$,

$$
f_{i}=\sum_{n=0}^{\infty} f_{i}^{n}(r) \mid n>
$$

the $f_{i}^{n}$ must decay as $r \rightarrow \infty$. Let us expand each $f_{i}^{n}$ in powers of $1 / r$. This implies that the $t_{i}^{n}$ have an expansion in $1 / r$. We can then reorganize the Taylor series for $f_{i}$ in the following way:

$$
\begin{aligned}
f_{i} & =t_{i}^{0}+t_{i}^{2} y^{2}+t_{i}^{4} y^{4}+\ldots \\
& =\sum_{p} \frac{1}{r^{p}} \sum_{k} b_{k}^{p, i} \mid k>
\end{aligned}
$$

The $b_{k}^{p, i}$ are just some numbers which determine the collection of harmonics contributing to a given power $1 / r^{p}$. Note that the oscillator eigenstates depend only on the combination $r y^{2}$ so that $\sum_{k} b_{k}^{p, i} \mid k>$ is a power series in $r y^{2}$.

For example, suppose that the harmonic $\mid m>$ is the only harmonic with a non-zero coefficient in the sum $\sum_{k} b_{k}^{p, i} \mid k>$ for the $1 / r^{p}$ term of $f_{i}$. We stress that in general there can be many harmonics contributing to a given term, but for simplicity, let us assume there is just one. The Taylor series for $f_{i}$ must then contain the terms:

$$
\begin{aligned}
f_{i} & =b_{m}^{p, i} \frac{1}{r^{p}}\left(1+a_{1}^{(m)} y^{2}+\ldots+a_{m}^{(m)} y^{2 m}\right) e^{-r y^{2} / 2}+\ldots \\
& =b_{m}^{p, i} \frac{1}{r^{p}}\left(1+\left\{a_{1}^{(m)}-\frac{1}{2} r\right\} y^{2}+\left\{a_{2}^{(m)}-\frac{1}{2} r a_{1}^{(m)}+\frac{1}{2^{2} 2 !} r^{2}\right\} y^{4}+\ldots\right)+\ldots \\
& =b_{m}^{p, i} \frac{1}{r^{p}}\left(1-\frac{1}{2}(1+m) r y^{2}+\frac{1}{24}\left(3+4 m+2 m^{2}\right) r^{2} y^{4}+\ldots\right)+\ldots
\end{aligned}
$$

There are specific relations between the Taylor coefficients in (5.3). We want to impose relations of this kind on the $t_{i}$ to satisfy normalizability in the $y$-direction for each choice of $p$ in the $1 / r$ expansion. 


\subsection{The structure of the solution}

Our Taylor series solution is completely determined by the three functions $t_{1}^{0}, t_{3}^{0}$ and $t_{4}^{0}$. These three functions must obey equations (6) and (7) of (4.22). To ensure that $t_{4}^{0}$ is decaying, we see from (6) that $t_{3}^{0}$ must take the form,

$$
t_{3}^{0}=\frac{c_{1}}{r^{3}}+\ldots
$$

where omitted terms decay more rapidly. For a given choice of $p$ in the $1 / r$ expansion, we wish to extract the terms in each $t_{3}^{n}$ which contribute to $\sum_{k} b_{k}^{p, 3} \mid k>$. Again, this is just the statement that we can organize the Taylor series for $f_{3}$ so that,

$$
f_{3}=\frac{1}{r^{3}} \sum_{k} b_{k}^{3,3} \mid k>+\ldots
$$

for some coefficients $b_{k}^{3,3}$. This is formally true for any Taylor series. What is generally not true is that generic $b_{k}^{3,3}$ give a wavefunction normalizable in the $y$ direction. Heuristically, the norm of $f_{3}$ should be dominated by the leading term in the $1 / r$ expansion. If we compute the norm of $f_{3}$ under this assumption, we see that:

$$
\left|f_{3}\right|^{2} \sim \int r^{4} d r\left\{\left(\frac{c_{1}}{r^{3}}\right)^{2} \frac{1}{r^{2}} \sum_{k} \frac{\left|b_{k}^{3,3}\right|^{2}}{(2+2 k)}\right\}
$$

where we have taken the normalization of $\mid k>$ given in Appendix D.

By looking at (4.19), (4.20), (4.21) and (4.22), we can see what terms in the Taylor series for $f_{3}$ have the right structure to determine the $b_{k}^{3,3}$ coefficients of (5.5). To quickly answer this question, let us list some order of magnitude relations which follow from (4.22) for the perturbative expansion. All relations are given in terms of our 3 independent functions $t_{1}^{0}, t_{3}^{0}$ and $t_{4}^{0}$,

$$
\begin{aligned}
& \text { (1) } t_{5}^{0} \sim O\left(t_{3}^{0} / r^{2}+t_{4}^{0} / r^{4}\right) \\
& \text { (2) } t_{11}^{0} \sim O\left(t_{4}^{0} / r^{2}\right) \\
& \text { (3) } t_{7}^{0} \sim O\left(t_{4}^{0}\right) \\
& \text { (4) } t_{9}^{0} \sim O\left(t_{3}^{0} / r^{2}\right) \\
& \text { (5) } t_{10}^{0} \sim O\left(t_{1}^{0}+t_{3}^{0} / r^{2}\right)
\end{aligned}
$$


There are similar order of magnitude relations for $t_{i}^{2}$ from (4.19),

$$
\begin{aligned}
& \text { (1) } t_{7}^{2} \sim O\left(t_{3}^{0}\right), \\
& \text { (2) } t_{9}^{2} \sim O\left(t_{1}^{0} / r^{2}+t_{4}^{0}\right), \\
& \text { (3) } t_{11}^{2} \sim O\left(t_{3}^{0} / r^{2}+t_{1}^{0}\right), \\
& \text { (4) } t_{5}^{2} \sim O\left(t_{4}^{0}+t_{3}^{0} / r^{4}\right), \\
& \text { (5) } t_{3}^{2} \sim O\left(r^{2} t_{4}^{0}+t_{3}^{0} / r^{2}\right), \\
& \text { (6) } t_{4}^{2} \sim O\left(t_{3}^{0}+t_{4}^{0} / r^{2}\right), \\
& \text { (7) } t_{10}^{2} \sim O\left(t_{4}^{0}+t_{1}^{0} / r^{2}\right), \\
& \text { (8) } t_{1}^{2} \sim O\left(t_{4}^{0}+t_{1}^{0} / r^{2}\right) .
\end{aligned}
$$

It is easy to continue and list order of magnitude relations for $t_{i}^{4}$ from (4.20), and higher $t_{i}^{n}$ using (4.21). These relations are useful for easily determining which terms in $t_{i}^{n}$ are relevant for determining the oscillator coefficients $b_{k}^{p, i}$.

Returning to our specific case of $f_{3}$, we see that since $t_{3}^{0} \sim 1 / r^{3}$, the only terms in $t_{3}^{2}$ relevant for computing $b_{k}^{3,3}$ are those proportional to $1 / r^{2}$. Looking at $t_{3}^{2}$ from (5.7), we see that the only way to have a term contributing to the $\sum b_{k}^{3,3} \mid k>$ is if,

$$
t_{4}^{0}=\frac{c_{1}^{\prime}}{r^{4}}+\ldots
$$

It could be the case that $c_{1}^{\prime}=0$, which means that the $b_{k}^{3,3}$ sum up in such a way that the $y^{2} / r^{2}$ term in (5.5) vanishes. Actually if $c_{1}^{\prime}=0$, the situation is much worse: we can see after some work that the $b_{k}^{3,3}$ have to be chosen so that the $\left(r y^{2}\right)^{1+2 n}$ terms in the Taylor expansion of,

$$
\sum_{k} b_{k}^{3,3} \mid k>
$$

vanish for all $n$. The $\left(r y^{2}\right)^{2 n}$ terms are all proportional to $c_{1}$ and give the constraint,

$$
c_{1}\left(1+\frac{1}{8}\left(r y^{2}\right)^{2}+\frac{1}{348}\left(r y^{2}\right)^{4}+\frac{1}{46080}\left(r y^{2}\right)^{6}+\ldots\right)=\sum_{k} b_{k}^{3,3} \mid k>\text {. }
$$

However, the left hand side of (5.9) is the expansion of the lowest oscillator $\mid 0>$ with the $\left(r y^{2}\right)^{1+2 n}$ terms missing. Since $\mid 0>=e^{-r y^{2} / 2}$, we immediately see that the left hand side of (5.9) sums to the expression:

$$
\frac{1}{2}\left\{e^{-r y^{2} / 2}+e^{r y^{2} / 2}\right\}
$$


This is clearly not normalizable so $c_{1}^{\prime}$ is necessarily non-zero. We will use this kind of argument repeatedly to determine the asymptotic solution.

With $c_{1}^{\prime} \neq 0$, we get a modified constraint:

$$
c_{1}\left(1+\frac{1}{8}\left(r y^{2}\right)^{2}+\ldots\right)-c_{1}^{\prime}\left(\frac{1}{10}\left(r y^{2}\right)+\frac{1}{240}\left(r y^{2}\right)^{3}+\ldots\right)=\sum_{k} b_{k}^{3,3} \mid k>
$$

It is easy to see that the terms proportional to $c_{1}^{\prime}$ sum up to give, 3

$$
\frac{1}{10}\left\{e^{-r y^{2} / 2}-e^{r y^{2} / 2}\right\}
$$

In this case, it is completely clear that we only get a normalizable solution if we pick,

$$
c_{1}^{\prime}=5 c_{1}
$$

More generally, we will encounter the situation where we have a Taylor series of the form,

$$
\alpha \sum_{n} d_{n}\left(r y^{2}\right)^{2 n}+\alpha^{\prime} \sum_{n} d_{n}^{\prime}\left(r y^{2}\right)^{2 n+1}
$$

where we know both $d_{n}$ and $d_{n}^{\prime}$, and either $\alpha$ or $\alpha^{\prime}$. Let us assume we know $\alpha$ and we wish to determine the values of $\alpha^{\prime}$ for which the Taylor series is normalizable. If the Taylor series can be fitted by a finite number of oscillators for some $\alpha^{\prime}$ then that choice of $\alpha^{\prime}$ is unique. To see this, suppose there were two distinct choices of $\alpha^{\prime}$. We could take the difference between the two series to obtain a Taylor series proportional to,

$$
\sum_{n} d_{n}^{\prime}\left(r y^{2}\right)^{2 n+1}
$$

However, this series cannot be generated by any sum over a finite number of oscillators since all the $\left(r y^{2}\right)^{2 n}$ terms must vanish. Therefore the choice of $\alpha^{\prime}$ is unique.

From equation (6) of (4.22), we see that the $O\left(1 / r^{4}\right)$ term in $t_{4}^{0}$ induces an $O\left(1 / r^{6}\right)$ correction to $t_{3}^{0}$. This begins to suggest a perturbation expansion in $1 / r^{3}$, and indeed that seems to be the case. From $t_{4}^{2} \sim O\left(1 / r^{6}\right)$, we see that we need a $O\left(1 / r^{7}\right)$ correction to $t_{4}^{0}$ and so on. It is easy to add $t_{1}^{0}$ into the story: from the expression for $t_{1}^{2} \sim O\left(1 / r^{4}\right)$, we

3 To see this, let us introduce new variables $\left(r, u=r y^{2} / 2\right)$. In terms of these variables and simple redefinitions of the $f_{i}$, the equations of (4.7) can be put in a triangular form with respect to the grading induced by the $1 / r$ expansion. In doing this, we treat $u$ as independent of $r$. The resulting triangular system is exactly solvable in the $1 / r$ expansion. 
learn that $t_{1}^{0} \sim 1 / r^{5}$ at leading order. From (7) of (4.22), we see that $t_{1}^{0}$ mixes with the first correction to $t_{4}^{0}$ and so on. What we have uncovered is the minimal required form for the solution given $c_{1} \neq 0$. The choice of $c_{1}$ then determines the normalization of the wavefunction. The solution is given by the expansion:

$$
\begin{aligned}
& t_{3}^{0}=\frac{c_{1}}{r^{3}}+\frac{c_{2}}{r^{6}}+\frac{c_{3}}{r^{9}}+\ldots, \\
& t_{4}^{0}=\frac{c_{1}^{\prime}}{r^{4}}+\frac{c_{2}^{\prime}}{r^{7}}+\frac{c_{3}^{\prime}}{r^{10}}+\ldots, \\
& t_{1}^{0}=\frac{c_{1}^{\prime \prime}}{r^{5}}+\frac{c_{2}^{\prime \prime}}{r^{8}}+\frac{c_{3}^{\prime \prime}}{r^{11}}+\ldots
\end{aligned}
$$

It is interesting that the $t_{i}^{0}$ have an expansion in powers of $1 / r^{3}$. Restoring the coupling constant, we see that this is an expansion in $g^{2} / r^{3}$. This is the natural expansion parameter in the gauge theory. From the perspective of the effective action on the Coulomb branch, what we are doing is summing the effects of the metric and all higher derivative corrections on the vacuum state of the gauge theory.

The expansion (5.13) represents the minimal required terms in the solution. It is natural to ask whether other powers of $1 / r$ are possible. Equation (6) of (4.22) requires that,

$$
t_{4}^{0} \sim O\left(r^{2} t_{3}^{0}\right)
$$

except for the special case where $t_{3}^{0} \sim O\left(1 / r^{3}\right)$. Likewise, equation (7) of (4.22) requires that,

$$
t_{1}^{0} \sim O\left(r^{2} t_{4}^{0}+\frac{1}{r^{4}} t_{4}^{0}\right)
$$

again except for the case $t_{4}^{0} \sim O\left(1 / r^{4}\right)$. Let us be concrete: suppose $t_{3}^{0}$ has a term of order $O\left(1 / r^{4}\right)$. This requires $t_{4}^{0} \sim O\left(1 / r^{2}\right)$ and therefore $t_{1}^{0} \sim O(1)$, which is not possible. Suppose $t_{3}^{0}$ has a term of order $O\left(1 / r^{5}\right)$, which implies that $t_{4}^{0} \sim O\left(1 / r^{3}\right)$. In turn, we see that $t_{3}^{2} \sim O(1 / r)$ which requires that $t_{3}^{0}=\alpha / r^{2}$. This is impossible unless $\alpha=0$. In that case, the Taylor expansion of $f_{3}$ has terms of the schematic form:

$$
f_{3} \sim O\left(\frac{y^{2}}{r}\right)+O\left(r y^{6}\right)+\ldots
$$

As in our earlier discussion, it is not hard to check that this sum is not normalizable. This kind of argument extends to higher powers: suppose $t_{3}^{0}$ has a term of order $O\left(1 / r^{7}\right)$, which implies that $t_{4}^{0} \sim O\left(1 / r^{5}\right)$. From $t_{3}^{2} \sim O\left(1 / r^{3}\right)$, we require a $O\left(1 / r^{4}\right)$ correction to $t_{3}^{0}$ which brings us back to an earlier case. This kind of reasoning suggests that only those terms generated by the original $1 / r^{3}$ term in $t_{3}^{0}$ appear in the solution. This also agrees with our gauge theory intuition. We shall therefore restrict our attention to the terms of (5.13). 


\subsection{Supergravity and the leading terms of the solution}

So far, we have found that the leading order term of $f_{3}$ is proportional purely to the oscillator ground state $\mid 0>$. We also know all the leading powers for the $t_{i}^{0}$. A glance at Appendix $\mathrm{C}$ tells us that the dominant terms for large $r$ in the bound state $\Psi$ are $f_{3}, f_{4}$ and $f_{5}$. Note that on the flat directions where $y=0$, the only non-vanishing forms in $\Psi$ are those with coefficients $f_{3}, f_{4}$ and $f_{5}$. It is not hard to check that both $f_{3}$ and $f_{5}$ are also proportional to $\mid 0>$ at leading order. Recalling that $r=\left|x^{1}\right|$, we have learnt that these leading terms are given by,

$$
\begin{aligned}
& f_{3}=\frac{c_{1}}{r^{3}} \mid 0>+\ldots, \\
& x^{1} f_{4}=\frac{5 c_{1} x^{1}}{r^{4}} \mid 0>+\ldots, \\
& \left(x^{1}\right)^{2} f_{5}=\frac{5 c_{1}\left(x^{1}\right)^{2}}{r^{5}} \mid 0>+\ldots
\end{aligned}
$$

Although each term transforms very differently under $\operatorname{Spin}(5)$ in either the $\mathbf{1 , 5}$ or $\mathbf{1 4}$, each decays at the same rate at leading order.

We can compare what we have learned about the exact Taylor series solution with what we might expect from an effective Hamiltonian construction like the one given in [14]. To construct the approximate bound state, we note that the wavefunction is sharply localized near the flat directions for large $r$. The potential term (2.20) is dominated by the $r^{2} y^{2}$ term. We conclude that for large $r$, the wavefunction can be expanded in a harmonic oscillator basis, with higher oscillator modes suppressed by powers of $1 / r$. The approximate bound state wavefunction takes the form of a product: there is a wavefunction of the light vector multiplet degrees of freedom multiplied by the ground state for the massive hypermultiplet degrees of freedom. We can now construct the effective Hamiltonian governing the long distance physics in a $1 / r$ expansion. The hypermultiplet ground state is easily determined from (2.16) to have the form, 国

$$
r e^{-r y^{2} / 2} d v_{1} d v_{2}
$$

In the most primitive approximation where the effective interactions are cancelled to order $1 / r$, the effective Hamiltonian is $(1 / 2) p^{\mu} p^{\mu}$ and acts on (5.15) multiplied by some function

4 The natural decomposition of $x^{\mu}$ and $q_{i}$ into light and heavy variables at large $r$ makes the construction of the approximate ground state much simpler than the non-abelian D0-D0 theory considered in [14]. 
of $x$ and $d u$. In fact, to determine the leading decay, we should go to order $1 / r^{2}$. Even then there will be a degenerate set of approximate ground states.

However, this rough approximation is good enough for the purpose of comparison with the structure following from the Taylor series solution. Using the forms given in Appendix C, we see that the coefficients in (5.14) precisely conspire to give agreement with the approximate construction. The leading terms in $\Psi$ sum to give,

$$
\begin{aligned}
\Psi & =f_{3}\left|2,2>_{\mathbf{1}}+f_{4} x^{1}\right| 2,2>_{\mathbf{5}}^{1}+f_{5}\left(x^{1}\right)^{2} \mid 2,2>_{\mathbf{1 4}}^{11}+\ldots \\
& \sim \frac{10 c_{1}}{r^{3}} e^{-r y^{2} / 2}\left(d u_{1} d u_{2}-d u_{3} d u_{4}\right)\left(d v_{1} d v_{2}\right)+\ldots
\end{aligned}
$$

where we take $x^{1}$ positive. There are a number of strange features of this asymptotic solution. First note that the vacuum for the massive fermions $d v_{1} d v_{2}$ is not a single representation of $\operatorname{Spin}(5)$. Let us contrast this with the case of 2 D0-branes where the vacuum for the massive fermions transforms in a single representation, the 44, of $\operatorname{Spin}(9)$ [15, 17]. The appearance of three different representations, the $\mathbf{1 , 5}$ and $\mathbf{1 4}$, in the asymptotic solution really cries out for an interpretation both in terms of the DLCQ M theory 5-brane, and in terms of the supergravity solution for the D0-D4 bound state.

What this seems to suggest is that the massive degrees of freedom never really decouple at large $r$. Through the Spin(5) flavor symmetry, the vacuum always knows about the existence of massive degrees of freedom. This issue is intimately tied to uniqueness of the bound state: the statement that the bound state is unique involves knowledge about both long and short distance physics. Uniqueness however forces invariance under the full Spin(5) acting on both light and heavy degrees of freedom. That $d v_{1} d v_{2}$ is not an irreducible representation then requires particular combinations of $\operatorname{Spin}(5)$ representations for the light degrees of freedom. A deeper understanding of this issue is certainly in order.

\subsection{Beyond supergravity}

Let us return to our general solution (5.13). We determined $c_{1}^{\prime}$ in terms of $c_{1}$ in (5.11). How do we determine $c_{1}^{\prime \prime}$ ? The straightforward way to determine $c_{1}^{\prime \prime}$ is not particularly elegant. We can take the Taylor series for $f_{1}$ and impose normalizability in the $y$-direction,

$$
\begin{aligned}
f_{1} & =\left(\frac{c_{1}^{\prime \prime}}{r^{5}}\right)+\left(\frac{5 c_{1}}{4 r^{4}}\right) y^{2}+\left(\frac{c_{1}^{\prime \prime}-10 c_{1}}{40 r^{3}}\right) y^{4}+\left(\frac{5 c_{1}}{96 r^{2}}\right) y^{6} \\
& +\left(\frac{c_{1}^{\prime \prime}}{4480 r}-\frac{c_{1}}{168 r}\right) y^{8}+O\left(y^{10}\right)+\ldots
\end{aligned}
$$


Note the general structure: half the terms in the Taylor series are known. The other half depend on the unknown constant that we wish to determine. It is not hard to see that the $\left(r y^{2}\right)^{1+2 n}$ terms come from expanding the lowest oscillator $\mid 0>$ state. The situation is then essentially the same as in (5.10), and we find that:

$$
c_{1}^{\prime \prime}=-\frac{5}{2} c_{1}
$$

From equation (6) of $(4.22)$, we find that $c_{2}$ is determined by $c_{1}^{\prime}$ :

$$
c_{2}=c_{1}
$$

Equation (7) of (4.22) relates $c_{2}^{\prime}$ to $c_{1}^{\prime \prime}$,

$$
c_{2}^{\prime}=-\frac{25}{2} c_{1}
$$

We are again back to the question of studying $f_{1}$ to determine $c_{2}^{\prime \prime}$. The relevant terms in $f_{1}$ begin at order $1 / r^{8}$,

$$
\begin{aligned}
f_{1}= & \frac{1}{r^{8}}\left(c_{2}^{\prime \prime}+\frac{125 c_{1}}{16}\left(r y^{2}\right)+\frac{c_{2}^{\prime \prime}-25 c_{1}}{40}\left(r y^{2}\right)^{2}+\frac{25 c_{1}}{384}\left(r y^{2}\right)^{3}\right. \\
& \left.+\left\{\frac{c_{2}^{\prime \prime}}{4480}+\frac{5 c_{1}}{384}\right\}\left(r y^{2}\right)^{4}+O\left(r y^{2}\right)^{5}\right)+\ldots
\end{aligned}
$$

Apart from more complicated coefficients, the general pattern is the same. Half the terms in the Taylor series are proportional to $c_{1}$ while the other half depend on the unknown constant $c_{2}^{\prime \prime}$. We can try to match (5.21) with an oscillator expansion involving a finite number of oscillators. Indeed, for the choice

$$
c_{2}^{\prime \prime}=-\frac{175}{8} c_{1}
$$

we can fit (5.21) by the first two oscillators:

$$
f_{1}=-\frac{225}{8} \frac{c_{1}}{r^{8}}\left(\left|0>-\frac{2}{9}\right| 1>\right)+\ldots
$$

As we might expect, higher excited oscillator states appear in the solution as we study more rapidly decreasing terms. This also suggests that only a finite number of oscillators will appear at any given order in the $1 / r$ expansion. 
Let us iterate the argument one more time. From (6) and (7) of (4.22), we immediately obtain the following relations:

$$
\begin{aligned}
& c_{3}=\frac{35}{2} c_{1}, \\
& c_{3}^{\prime}=-\frac{195}{2} c_{1} .
\end{aligned}
$$

The relevant terms in $f_{1}$ take the form,

$$
\begin{aligned}
f_{1}= & \frac{1}{r^{11}}\left(c_{3}^{\prime \prime}+\frac{2825 c_{1}}{16}\left(r y^{2}\right)+\left\{\frac{c_{3}^{\prime \prime}}{40}-\frac{595 c_{1}}{32}\right\}\left(r y^{2}\right)^{2}\right. \\
& \left.+\frac{425 c_{1}}{128}\left(r y^{2}\right)^{3}+O\left(r y^{2}\right)^{4}\right)+\ldots
\end{aligned}
$$

and can be fit by the first three oscillator modes,

$$
f_{1}=-\frac{525}{32} \frac{c_{1}}{r^{11}}\left(\left|2>-\frac{208}{21}\right| 1>+\frac{115}{3} \mid 0>\right)+\ldots
$$

for the choice,

$$
c_{3}^{\prime \prime}=-\frac{7725}{16} c_{1}
$$

\section{A Remarkable Reduction}

\subsection{Prolongation}

Studying the bound state solution in an asymptotic series is analogous to studying the M5-brane in a derivative expansion around the supergravity solution. Ideally, we want a more powerful technique to solve the vacuum equations. The aim of this final section is to present a more global approach to solving the vacuum equations. Hopefully, this approach is closer to the method an $\mathrm{M}$ theorist might use to study the M5-brane.

We shall present a surprising reduction of the long list of equations (4.7) to a single scalar elliptic equation. The equation takes the form,

$$
(\Delta+\vec{B} \cdot \nabla+W) u=0
$$

where $\Delta=\partial_{r}^{2}+\partial_{y}^{2}$, the vector field $\vec{B}$ with components $\left(B_{r}, B_{y}\right)$ and the potential $W$ are rational functions of $r$ and $y$. The function $u=u(r, y)$ is a particular combination of the $f_{i}$. In order to explain how we reduce our first order system to a single second 
order equation, let us first consider the inverse process: prolongation. To understand the procedure, let us begin with an illustrative example: we take an equation of the form,

$$
F_{x x}+F_{y y}+U F=0 .
$$

We can define new functions $p=F_{x}$ and $q=F_{y}$ with which we can 'prolong' our scalar second order equation into a system of first order equations:

$$
\begin{aligned}
& p_{x}+q_{y}+U F=0, \\
& F_{x}=p, \\
& F_{y}=q .
\end{aligned}
$$

We also have a compatibility relation,

$$
F_{x y}=F_{y x}
$$

which implies an additional fourth equation:

$$
p_{y}-q_{x}=0
$$

We can express these relations as a differential system,

$$
\begin{aligned}
& d F=p d x+q d y, \\
& d p=a d x+b d y, \\
& d q=b d x-(a+U F) d y .
\end{aligned}
$$

We could repeat the procedure and prolong again by adding the equations,

$$
\begin{aligned}
& d a=c d x+e d y \\
& d b=e d x+\left((U F)_{x}-c\right) d y
\end{aligned}
$$

Each time we prolong a system of equations like this, we add two new unknown functions. These functions are the unknown derivatives of the functions comprising the previous system of equations. 


\subsection{Deprolongation}

Now we would like to 'deprolong' our system of equations (4.7). 5 We shall see that the system (4.7) containing 7 independent functions can be obtained by prolonging a single second order equation three times. In order to deprolong (4.7), we write the equations as an exterior system as before. We choose 5 functions $F_{i}$ which are linear combinations of the initial seven $f_{i}$ so that $d F_{i}$ is expressible algebraically in terms of the original seven. We keep the 5 equations defining the $d F_{i}$ and discard the remaining two equations. For example, for this first step, we can make the following choice:

$$
\begin{gathered}
F_{1}=f_{1}+f_{10}, \\
F_{2}=r^{2} f_{5}-y^{2} f_{1}, \\
F_{3}=f_{7}, \quad F_{4}=f_{4}, \quad F_{5}=f_{11} .
\end{gathered}
$$

So far the equations remain first order. We then iterate this procedure until we arrive at the first prolongation of the scalar equation. We then make the obvious substitution to transform the first prolongation into a second order scalar equation.

Let us summarize the results of the deprolongation. We obtain the following equation:

$$
u_{r r}+u_{y y}+\frac{\widetilde{B}_{r}}{r F} u_{r}+\frac{\widetilde{B}_{y}}{y F} u_{y}+\frac{\widetilde{W}}{F} u=0 .
$$

We define $u$ in terms of the variables $s=y^{2} / 2$ and $t=r^{2} / 2$ and the functions $f_{1}, f_{4}, f_{5}, f_{7}, f_{10}, f_{11}$ :

$$
\begin{aligned}
u= & (2 s-t) f_{7}-\left(\frac{4}{3} t s^{2}-\frac{1}{3} t^{2} s-\frac{1}{6} t^{3}-1\right) f_{10}+\left(\frac{4}{3} t s^{2}-\frac{1}{3} t^{2} s-\frac{1}{6} t^{3}+1\right) f_{1} \\
& -s f_{4}+\left(\frac{2}{3} t s^{2}-\frac{8}{3} s^{3}+\frac{1}{3} t^{2} s\right) f_{5}-\left(\frac{2}{3} t s-\frac{8}{3} s^{2}+\frac{5}{6} t^{2}\right) f_{11} .
\end{aligned}
$$

It would be interesting to find a geometric interpretation for (6.7) - perhaps in terms of some bundle over either $\mathbb{R}^{2}$ or $\mathbb{R}^{9}$. Let us list the rational functions which appear in (6.7). For $\widetilde{B}_{r}$, we find the expression:

$$
\begin{aligned}
\widetilde{B}_{r}= & -224 t^{3} s^{3}+576 t^{2} s^{4}+32 t^{4} s^{2}-12 t^{5} s+4 t^{6}-12 t^{2} s \\
& +144 t s^{2}-24 t^{3}-448 s^{5} t-48 s^{3}+36
\end{aligned}
$$

5 We are especially grateful to Robert Bryant for suggesting and explaining this reduction to us. 
while $\widetilde{B}_{y}$ and $F$ are given by,

$$
\begin{aligned}
\widetilde{B}_{y} & =272 t^{2} s^{4}-192 t^{3} s^{3}-40 t^{4} s^{2}+264 t s^{2}-216 t^{2} s+68 t^{5} s+42 t^{3}-15 t^{6}+9-64 s^{5} t \\
F & =9+64 t^{3} s^{3}-8 t^{4} s^{2}-6 t^{3}+24 t^{2} s-112 t^{2} s^{4}+t^{6}-24 t s^{2}+64 s^{5} t-4 t^{5} s
\end{aligned}
$$

Lastly, the function $\widetilde{W}$ which determines the potential $W$ takes the form:

$$
\begin{aligned}
\widetilde{W}= & -105 t^{2}+80 s^{3} t^{2}+38 t^{5}+144 s t-96 t^{3} s^{2}+24 s^{2}-144 t^{4} s^{4}-32 t^{5} s^{3} \\
& +24 t^{6} s^{2}-256 s^{6} t^{2}+224 s^{4} t+384 s^{5} t^{3}-t^{8}-76 s t^{4} .
\end{aligned}
$$

Equation (6.7) is remarkably simple by comparision with (4.7). That the equations reduce this way opens up the possibility of answering a host of otherwise intractable questions.

\section{Acknowledgements}

It is our pleasure to thank Robert Bryant for suggesting and explaining deprolongation which led to the results of section six. The work of S.S. is supported by the William Keck Foundation and by NSF grant PHY-9513835; that of M.S. by NSF grant DMS-9870161. 


\section{Appendix A. Quaternions and Symplectic Groups}

We will summarize some useful relations between quaternions and symplectic groups. Let us label a basis for our quaternions by $\{\mathbf{1}, I, J, K\}$ where,

$$
I^{2}=J^{2}=K^{2}=-\mathbf{1}, \quad I J K=-\mathbf{1} .
$$

A quaternion $q$ can then be expanded in components

$$
q=q^{1}+I q^{2}+J q^{3}+K q^{4}
$$

The conjugate quaternion $\bar{q}$ has an expansion

$$
q=q^{1}-I q^{2}-J q^{3}-K q^{4}
$$

The symmetry group $S p(1)_{R} \sim S U(2)_{R}$ is the group of unit quaternions. Let $\Lambda$ be a field transforming in the $\mathbf{2}$ of $S p(1)_{R}$. If we view $S p(1)_{R}$ acting on $\Lambda$ as right multiplication by a unit quaternion $g$ then,

$$
\Lambda \rightarrow \Lambda g
$$

In this formalism, $\Lambda$ is valued in the quaternions. Equivalently, we can expand $\Lambda$ in components and express the action of $g$ in the following way,

$$
\Lambda_{a} \rightarrow g_{a b} \Lambda_{b}
$$

where $g_{a b}$ implements right multiplication by the unit quaternion $g$. For example, right multiplication by $I$ on $q$ gives

$$
\begin{aligned}
q & \rightarrow q I \\
& \rightarrow q^{1} I-q^{2}-q^{3} K+q^{4} J
\end{aligned}
$$

which can be realized by the matrix

$$
I^{R}=\left(\begin{array}{cccc}
0 & -1 & 0 & 0 \\
1 & 0 & 0 & 0 \\
0 & 0 & 0 & 1 \\
0 & 0 & -1 & 0
\end{array}\right)
$$

acting on $q$ in the usual way $q_{a} \rightarrow I_{a b}^{R} q_{b}$. The matrices $J^{R}$ and $K^{R}$ realize right multiplication by $J, K$ while $\mathbf{1}^{R}$ is the identity matrix:

$$
J^{R}=\left(\begin{array}{cccc}
0 & 0 & -1 & 0 \\
0 & 0 & 0 & -1 \\
1 & 0 & 0 & 0 \\
0 & 1 & 0 & 0
\end{array}\right), \quad K^{R}=\left(\begin{array}{cccc}
0 & 0 & 0 & -1 \\
0 & 0 & 1 & 0 \\
0 & -1 & 0 & 0 \\
1 & 0 & 0 & 0
\end{array}\right) .
$$


We define operators $s^{j}$ in terms of $\left\{\mathbf{1}^{R}, I^{R}, J^{R}, K^{R}\right\}$

$$
s^{1}=\left(\begin{array}{cc}
\mathbf{1}^{R} & 0 \\
0 & \mathbf{1}^{R}
\end{array}\right), \quad s^{2}=\left(\begin{array}{cc}
I^{R} & 0 \\
0 & I^{R}
\end{array}\right), \quad s^{3}=\left(\begin{array}{cc}
J^{R} & 0 \\
0 & J^{R}
\end{array}\right), \quad s^{4}=\left(\begin{array}{cc}
K^{R} & 0 \\
0 & K^{R}
\end{array}\right) .
$$

In a similar way, the group $S p(2) \sim \operatorname{Spin}(5)$ is the group of quaternion-valued $2 \times 2$ matrices with unit determinant. We will view $S p(2)$ as acting by left multiplication on a field $\Psi$ in the defining representation. So an element $U \in S p(2)$ acts in the following way:

$$
\Psi \rightarrow U \Psi
$$

Equivalently, in terms of components

$$
\Psi_{a} \rightarrow U_{a b} \Psi_{b}
$$

Lastly, we can give an explicit form for the gamma matrices (2.3) in terms of quaternions:

$$
\begin{gathered}
\gamma^{1}=\left(\begin{array}{cc}
1 & 0 \\
0 & -1
\end{array}\right), \quad \gamma^{2}=\left(\begin{array}{ll}
0 & 1 \\
1 & 0
\end{array}\right), \quad \gamma^{3}=\left(\begin{array}{cc}
0 & I \\
-I & 0
\end{array}\right) \\
\gamma^{4}=\left(\begin{array}{cc}
0 & J \\
-J & 0
\end{array}\right), \quad \gamma^{5}=\left(\begin{array}{cc}
0 & K \\
-K & 0
\end{array}\right) .
\end{gathered}
$$

In turn, $\{I, J, K\}$ can be expressed in terms of the Pauli matrices $\sigma^{i}$

$$
\sigma^{1}=\left(\begin{array}{cc}
0 & 1 \\
1 & 0
\end{array}\right), \quad \sigma^{2}=\left(\begin{array}{cc}
0 & -i \\
i & 0
\end{array}\right), \quad \sigma^{3}=\left(\begin{array}{cc}
1 & 0 \\
0 & -1
\end{array}\right)
$$

as $4 \times 4$ real anti-symmetric matrices:

$$
I=\left(\begin{array}{cc}
0 & \sigma^{1} \\
-\sigma^{1} & 0
\end{array}\right), \quad J=\left(\begin{array}{cc}
-i \sigma^{2} & 0 \\
0 & -i \sigma^{2}
\end{array}\right), \quad K=\left(\begin{array}{cc}
0 & \sigma^{3} \\
-\sigma^{3} & 0
\end{array}\right) .
$$




\section{Appendix B. Forms and Representations of $S p(2)$}

Using the complexification (3.7), we obtain a set of Hermitian $4 \times 4$ matrices from those given in Appendix A:

$$
\begin{gathered}
\tilde{\gamma}^{1}=\left(\begin{array}{cc}
1 & 0 \\
0 & -1
\end{array}\right), \quad \tilde{\gamma}^{2}=\left(\begin{array}{ll}
0 & 1 \\
1 & 0
\end{array}\right), \quad \tilde{\gamma}^{3}=\left(\begin{array}{cc}
0 & -i \sigma^{1} \\
i \sigma^{1} & 0
\end{array}\right) \\
\tilde{\gamma}^{4}=\left(\begin{array}{cc}
0 & -i \sigma^{3} \\
i \sigma^{3} & 0
\end{array}\right), \quad \tilde{\gamma}^{5}=\left(\begin{array}{cc}
0 & i \sigma^{2} \\
-i \sigma^{2} & 0
\end{array}\right) .
\end{gathered}
$$

We also need the symplectic metric or charge conjugation matrix,

$$
C=\left(\begin{array}{cccc}
0 & 1 & 0 & 0 \\
-1 & 0 & 0 & 0 \\
0 & 0 & 0 & 1 \\
0 & 0 & -1 & 0
\end{array}\right)
$$

which implements complex conjugation:

$$
C \tilde{\gamma} C=-\tilde{\gamma}^{*} .
$$

Our forms $d u_{a}$ and $d v_{a}$ transform in the $\mathbf{4}$ of $S p(2)$. The representation $\wedge^{2} \mathbf{4}$ decomposes into $\mathbf{5} \oplus \mathbf{1}$. As an example, we can explicitly construct the $\mathbf{1}$ from $(1,1)$ forms in the following way,

$$
d u C d v
$$

while the $\mathbf{5}$ is given by:

$$
d u \tilde{\gamma}^{\mu} C d v
$$

It is not hard to check that these combinations transform correctly. Lastly, we need to consider $\mathbf{5} \otimes \mathbf{5}=\mathbf{1} \oplus \mathbf{1 0} \oplus \mathbf{1 4}$ since the $\mathbf{1}$ and the $\mathbf{1 4}$ appear in (3.14). The $\mathbf{1}$ is given by the form,

$$
\sum_{\mu} d u \tilde{\gamma}^{\mu} C d u d v \tilde{\gamma}^{\mu} C d v
$$

while the $\mathbf{1 4}$ has components:

$$
d u \tilde{\gamma}^{(\mu} C d u d v \tilde{\gamma}^{\nu)} C d v-\frac{1}{5} \delta^{\mu \nu} \sum_{\rho} d u \tilde{\gamma}^{\rho} C d u d v \tilde{\gamma}^{\rho} C d v
$$




\section{Appendix C. A Word on Normalizations}

To fix the choice of normalizations, we list explicitly the forms appearing in (3.12) through (3.18) at the special point where $x^{1} \neq 0$ with $x^{\mu}=0$ for $\mu>1$ and $q_{1} \neq 0$ with $q_{j}=0$ for $j>1$. All forms act on the canonical vacuum $\mid 0>$ which is omitted,

$$
\begin{aligned}
& f_{1} \mid 0,0>=f_{1}\left(q_{1}\right)^{2} d u_{1} d u_{2} d u_{3} d u_{4}, \\
& f_{2} \mid 0,4>=f_{2}\left(q_{1}\right)^{2} d v_{1} d v_{2} d v_{3} d v_{4} \\
& f_{3} \mid 2,2>_{\mathbf{1}}=f_{3}\left\{\left(d u_{1} d u_{2}-d u_{3} d u_{4}\right)\left(d v_{1} d v_{2}-d v_{3} d v_{4}\right)-\right. \\
& 2\left(d u_{1} d u_{4} d v_{2} d v_{3}+d u_{2} d u_{3} d v_{1} d v_{4}\right)+ \\
& \left.2\left(d u_{1} d u_{3} d v_{2} d v_{4}+d u_{2} d u_{4} d v_{1} d v_{3}\right)\right\} \\
& f_{4} x^{1} \mid 2,2>_{\mathbf{5}}^{1}=f_{4} x^{1}\left(d u_{1} d u_{2}-d u_{3} d u_{4}\right)\left(d v_{1} d v_{2}+d v_{3} d v_{4}\right), \\
& f_{5}\left(x^{1}\right)^{2} \mid 2,2>_{\mathbf{1 4}}^{11}=f_{5}\left(x^{1}\right)^{2}\left\{\frac{4}{5}\left(d u_{1} d u_{2}-d u_{3} d u_{4}\right)\left(d v_{1} d v_{2}-d v_{3} d v_{4}\right)+\right. \\
& \frac{2}{5}\left(d u_{1} d u_{4} d v_{2} d v_{3}+d u_{2} d u_{3} d v_{1} d v_{4}\right)- \\
& \left.\frac{2}{5}\left(d u_{1} d u_{3} d v_{2} d v_{4}+d u_{2} d u_{4} d v_{1} d v_{3}\right)\right\} \\
& f_{6} \mid 1,3>=f_{6} q_{1}\left(d v_{1} d v_{2}+d v_{3} d v_{4}\right)\left(d u_{1} d v_{2}-d u_{2} d v_{1}+d u_{3} d v_{4}-d u_{4} d v_{3}\right), \\
& f_{7} \mid 1,1>=f_{7} q_{1}\left(d u_{1} d u_{2}+d u_{3} d u_{4}\right)\left(d u_{1} d v_{2}-d u_{2} d v_{1}+d u_{3} d v_{4}-d u_{4} d v_{3}\right), \\
& f_{8} x^{1} \mid 1,3>^{1}=f_{8} x^{1} q_{1}\left(d v_{1} d v_{2}+d v_{3} d v_{4}\right)\left(d u_{1} d v_{2}-d u_{2} d v_{1}-d u_{3} d v_{4}+d u_{4} d v_{3}\right), \\
& f_{9} x^{1} \mid 1,1>^{1}=f_{9} x^{1} q_{1}\left(d u_{1} d u_{2}+d u_{3} d u_{4}\right)\left(d u_{1} d v_{2}-d u_{2} d v_{1}-d u_{3} d v_{4}+d u_{4} d v_{3}\right), \\
& f_{10} \mid 0,2>=f_{10} \frac{1}{2}\left(q_{1}\right)^{2}\left(d u_{1} d u_{2}+d u_{3} d u_{4}\right)\left(d v_{1} d v_{2}+d v_{3} d v_{4}\right) \\
& f_{11} x^{1} \mid 0,2>^{1}=f_{11} x^{1} \frac{1}{2}\left(q_{1}\right)^{2}\left(d u_{1} d u_{2}+d u_{3} d u_{4}\right)\left(d v_{1} d v_{2}-d v_{3} d v_{4}\right) \text {. }
\end{aligned}
$$




\section{Appendix D. The Four-Dimensional Radial Harmonic Oscillator}

We want to construct eigenstates for the radial four-dimensional simple harmonic oscillator which satisfy,

$$
\left\{-\partial_{y}^{2}-\frac{3}{y} \partial_{y}+r^{2} y^{2}\right\}\left|n>=E_{n}\right| n>
$$

where $E_{n}=4(n+1) r$. The easiest is the ground state:

$$
\mid 0>=e^{-r y^{2} / 2}
$$

A general eigenstate takes the form,

$$
\mid n>=\left(1+a_{1}^{(n)} y^{2}+\ldots+a_{n}^{(n)} y^{2 n}\right) e^{-r y^{2} / 2} .
$$

It is not hard to check using the nice relation,

$$
\left\{-\partial_{y}^{2}-\frac{3}{y} \partial_{y}+r^{2} y^{2}\right\} y^{2 n} e^{-r y^{2} / 2}=\left\{E_{n} y^{2 n}-4 n(n+1) y^{2 n-2}\right\} e^{-r y^{2} / 2},
$$

that each coefficient $a_{m}^{(n)}$ is determined by the recursion relation,

$$
a_{m}^{(n)}=\frac{a_{m-1}^{(n)}(m-n-1) r}{m(m+1)},
$$

where $a_{0}^{(n)}=1$. Note that these eigenstates are not normalized, but they are orthogonal when integrated with the measure $y^{3} d y$. The norm of these eigenstates is given by the formula,

$$
<n \mid n>=\frac{1}{r^{2}} \frac{1}{(2+2 n)} .
$$

We will need to evaluate various operators acting on $|n\rangle$. The nicest are the three operators $y^{2}, y \partial_{y}, \partial_{r}$. These operators raise and lower by at most one unit:

$$
\begin{aligned}
r y^{2} \mid n> & =-n|n-1>+2(n+1)| n>-(n+2) \mid n+1>, \\
y \partial_{y} \mid n> & =-n|n-1>-2| n>+(n+2) \mid n+1>, \\
2 r \partial_{r} \mid n> & =-n|n-1>-2| n>+(n+2) \mid n+1>.
\end{aligned}
$$

Note that $y \partial_{y}$ is equivalent to $2 r \partial_{r}$ when acting on $|n\rangle$. 


\section{Appendix E. Equations from a Taylor Expansion}

We list explicitly the equations that follow from a Taylor expansion of the $f_{i}$ in the $y$-direction and which only involve $t_{i}^{0}$ and $t_{i}^{2}$,
(1) $\frac{1}{2} t_{1}^{0}-\frac{r^{2}}{5} t_{5}^{2}+t_{11}^{2}+t_{3}^{2}=0$
(2) $r \partial_{r} t_{9}^{2}-6 t_{1}^{2}-\frac{1}{2} t_{7}^{0}+5 t_{9}^{2}+2 t_{10}^{2}=0$,
(3) $\partial_{r} t_{7}^{2}+2 r t_{11}^{2}+r\left\{t_{1}^{0}-\frac{1}{2} t_{9}^{0}\right\}=0$,
(4) $2 r t_{9}^{2}+\partial_{r} t_{1}^{0}+r t_{7}^{0}=0$,
(5) $2 t_{7}^{2}+r^{2} t_{9}^{0}=0$,
(6) $4 t_{10}^{2}-4 t_{1}^{2}+5 t_{9}^{2}+r^{2} f_{11}^{0}+r \partial_{r} t_{9}^{2}+\frac{1}{2} t_{7}^{0}=0$,
(7) $2 r^{2} t_{4}^{0}+3 t_{9}^{0}-r \partial_{r} t_{9}^{0}+4 t_{3}^{2}+\frac{16}{5} r^{2} t_{5}^{2}=0$,
(8) $\frac{4}{5} r^{2} t_{5}^{2}-4 t_{3}^{2}-r \partial_{r} t_{9}^{0}-3 t_{9}^{0}=0$,
(9) $t_{10}^{0}+\frac{1}{2} t_{9}^{0}+4 t_{11}^{2}+\frac{1}{r} \partial_{r} t_{7}^{2}=0$
(10) $2 t_{3}^{0}+\frac{8}{5} r^{2} t_{5}^{0}+4 t_{4}^{2}-\frac{1}{r} \partial_{r} t_{7}^{0}=0$,
(11) $r\left\{t_{4}^{0}+t_{7}^{0}\right\}-2 r t_{9}^{2}+\partial_{r} t_{10}^{0}=0$,
(12) $\frac{56}{5} r t_{5}^{2}-r t_{7}^{0}+6 r t_{9}^{2}+2 \partial_{r} t_{3}^{2}+\frac{8}{5} r^{2} \partial_{r} t_{5}^{2}=0$,
(13) $\frac{2}{r} \partial_{r} t_{3}^{2}-\frac{2}{5} r \partial_{r} t_{5}^{2}-\frac{14}{5} t_{5}^{2}-t_{7}^{0}-6 t_{9}^{2}=0$,
(14) $10 t_{4}^{2}+6 t_{7}^{2}-r^{2} t_{9}^{0}+2 r \partial_{r} t_{4}^{2}=0$,
(15) $t_{3}^{0}+\frac{4}{5} r^{2} t_{5}^{0}+r^{2} t_{9}^{0}+t_{11}^{0}-2 t_{7}^{2}+r \partial_{r} t_{11}^{0}=0$. 
Those equations that only involve $t_{i}^{0}, t_{i}^{2}$ and $t_{i}^{4}$ are given below:
(1) $t_{3}^{4}+\frac{1}{2} t_{1}^{2}-\frac{r^{2}}{5} t_{5}^{4}+t_{11}^{4}=0$,
(2) $r \partial_{r} t_{9}^{4}-8 t_{1}^{4}-\frac{1}{2} t_{7}^{2}+5 t_{9}^{4}+2 t_{10}^{4}=0$,
(3) $\partial_{r} t_{7}^{4}+2 r t_{11}^{4}+r\left\{t_{1}^{2}-\frac{1}{2} t_{9}^{2}\right\}=0$,
(4) $4 r t_{9}^{4}+\partial_{r} t_{1}^{2}+r t_{7}^{2}=0$,
(5) $4 t_{7}^{4}+r^{2} t_{9}^{2}+\frac{1}{2} t_{1}^{0}=0$,
(6) $6 t_{10}^{4}-4 t_{1}^{4}+5 t_{9}^{4}+r^{2} f_{11}^{2}+r \partial_{r} t_{9}^{4}+\frac{1}{2} t_{7}^{2}=0$,
(7) $2 r^{2} t_{4}^{2}-\frac{1}{2} t_{7}^{0}+3 t_{9}^{2}-r \partial_{r} t_{9}^{2}+8 t_{3}^{4}+\frac{32}{5} r^{2} t_{5}^{4}=0$,
(8) $\frac{8}{5} r^{2} t_{5}^{4}-8 t_{3}^{4}-r \partial_{r} t_{9}^{2}-3 t_{9}^{2}+\frac{1}{2} t_{7}^{0}=0$,
(9) $t_{10}^{2}+\frac{1}{2} t_{9}^{2}+6 t_{11}^{4}+\frac{1}{r} \partial_{r} t_{7}^{4}=0$,
(10) $2 t_{3}^{2}+\frac{8}{5} r^{2} t_{5}^{2}-\frac{1}{2} t_{9}^{0}+8 t_{4}^{4}-\frac{1}{r} \partial_{r} t_{7}^{2}=0$,
(11) $r\left\{t_{4}^{2}+t_{7}^{2}\right\}-4 r t_{9}^{4}+\partial_{r} t_{10}^{2}=0$,
(12) $\frac{56}{5} r t_{5}^{4}-r t_{7}^{2}+8 r t_{9}^{4}+\frac{r}{2} t_{11}^{0}+2 \partial_{r} t_{3}^{4}+\frac{8}{5} r^{2} \partial_{r} t_{5}^{4}=0$,
(13) $\frac{2}{r} \partial_{r} t_{3}^{4}-\frac{2}{5} r \partial_{r} t_{5}^{4}-\frac{14}{5} t_{5}^{4}-t_{7}^{2}-8 t_{9}^{4}=0$,
(14) $10 t_{4}^{4}+8 t_{7}^{4}-r^{2} t_{9}^{2}+\frac{1}{2} t_{10}^{0}+2 r \partial_{r} t_{4}^{4}=0$,
(15) $t_{3}^{2}+\frac{4}{5} r^{2} t_{5}^{2}+r^{2} t_{9}^{2}+t_{11}^{2}-4 t_{7}^{4}+r \partial_{r} t_{11}^{2}=0$. 


\section{References}

[1] S. Sethi and M. Stern, hep-th/9607145, Phys. Lett. B398 (1997), 47.

[2] M. Berkooz and M. R. Douglas, hep-th/9610236, Phys. Lett. B395 (1997) 196.

[3] E. Witten, hep-th/9511030, Nucl. Phys. B460 (1996) 541.

[4] M. R. Douglas, D. Kabat, P. Pouliot and S. Shenker, hep-th/9608024, Nucl. Phys. B485 (1997), 85.

[5] D.-E. Diaconescu and R. Entin, hep-th/9706059, Phys. Rev. D56 (1997) 8045.

[6] S. Sethi and M. Stern, hep-th/0001189.

[7] S. Paban, S. Sethi and M. Stern, hep-th/9805018, Nucl. Phys. B534 (1998) 137.

[8] A. A. Tseytlin, hep-th/9604035, Nucl. Phys. B475 (1996) 149.

[9] T. Banks, W. Fischler, S. H. Shenker, and L. Susskind, hep-th/9610043, Phys. Rev. D55 (1997) 5112.

[10] A. Connes, M. R. Douglas and A. Schwarz, hep-th/9711162, JHEP 9802:003, 1998.

[11] E. Keski-Vakkuri and P. Kraus, hep-th/9712013, Nucl. Phys. B529 (1998) 246.

[12] S. P. de Alwis, hep-th/9806178, Phys. Rev. D59 (1999) 044029.

[13] K. Becker and M. Becker, hep-th/9712238, Phys. Rev. D57 (1998) 6464.

[14] S. Sethi and M. Stern, hep-th/9705046, Comm. Math. Phys. 194 (1998) 675.

[15] M. Halpern and C. Schwartz, hep-th/9712133, Int. J. Mod. Phys. A13 (1998) 4367.

[16] J. Plefka and A. Waldron, hep-th/9710104, Nucl. Phys. B512 (1998) 460.

[17] J. Frohlich, G. M. Graf, D. Hasler, J. Hoppe and S.-T. Yau, hep-th/9904182.

[18] P. Yi, hep-th/9704098, Nucl. Phys. B505 (1997) 307. 